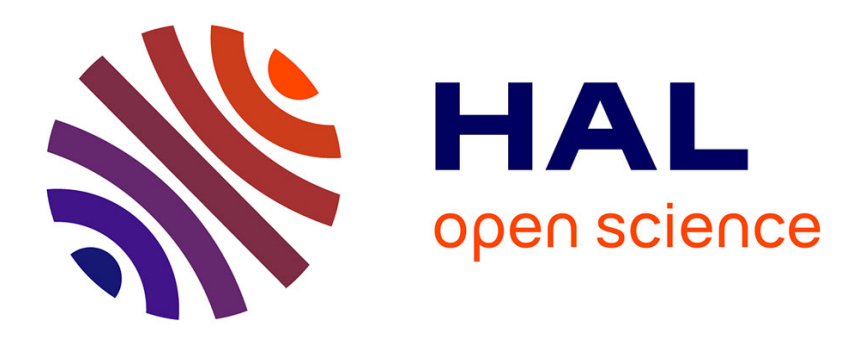

\title{
Propagation of spike sequences in neural networks
}

Arnaud Tonnelier

\section{To cite this version:}

Arnaud Tonnelier. Propagation of spike sequences in neural networks. SIAM Journal on Applied Dynamical Systems, 2010, 9 (3), pp.1090-1118. 10.1137/100782504 . hal-00525420

\section{HAL Id: hal-00525420 \\ https://hal.science/hal-00525420}

Submitted on 11 Oct 2010

HAL is a multi-disciplinary open access archive for the deposit and dissemination of scientific research documents, whether they are published or not. The documents may come from teaching and research institutions in France or abroad, or from public or private research centers.
L'archive ouverte pluridisciplinaire HAL, est destinée au dépôt et à la diffusion de documents scientifiques de niveau recherche, publiés ou non, émanant des établissements d'enseignement et de recherche français ou étrangers, des laboratoires publics ou privés. 


\title{
PROPAGATION OF SPIKE SEQUENCES IN NEURAL NETWORKS
}

\author{
ARNAUD TONNELIER*
}

\begin{abstract}
Precise spatiotemporal sequences of action potentials are observed in many brain areas and are thought to be involved in the neural processing of sensory stimuli. Here, we examine the ability of spiking neural networks to propagate stably a spatiotemporal sequence of spikes in the limit where each neuron fires only one spike. In contrast to previous studies on propagation in neural networks, we assume only homogeneous connectivity and do not use the continuum approximation. When the propagation is associated with a simple traveling wave, or a one-spike sequence, we derive some analytical results for the wave speed and show that its stability is determined by the Schur criterion. The propagation of a sequence of several spikes corresponds to the existence of stable composite waves, i.e., stable spatiotemporal periodic traveling waves. The stability of composite waves is related to the roots of a system of multivariate polynomials. Using the simplest synaptic architecture that supports composite waves, a three nearest-neighbor coupling feedforward network, we analytically and numerically investigate the propagation of 2-composite waves, i.e., two-spike sequence propagation. The influence of the synaptic coupling, stochastic perturbations, and neuron parameters on the propagation of larger sequences is also investigated.
\end{abstract}

Key words. spiking neuron, propagation, traveling wave, spike sequence

AMS subject classifications. $92 \mathrm{C} 20$

1. Introduction. Precisely timed action potentials, or spikes, have been directly related to sensory stimuli, indicating a possible role in the neural processing of information $[2,25]$. A coding scheme based on the spikes emitted by neurons allows fast processing and direct experimental evidence of reliable and efficient spike coding strategies has been emphasized [39, 25]. The implementation of spike coding schemes has shown their ability to perform very rapid processing that precludes conventional firing rate codes [37]. The spikes emitted by different neurons in the network form a spatiotemporal pattern that is believed to be involved in the encoding of sensory stimuli. In the locust antennal lobe, the odor-evoked activity patterns of the projection neurons are structured spatially and temporally in a stimulus-specific manner [27]. In songbirds, the projection neurons in the premotor nucleus HVC (high vocal center) exhibit sequences of precise spike timings [18]. A code based on the precise firing sequence of spikes requires a means of propagating the sequence while preserving its temporal structure. The main question is thus whether a packet of precisely timed action potentials can be transmitted reliably by spiking neural networks.

A number of studies have examined propagating activities using neural field models $[32,7,33,38]$. In spiking neural networks, the information is encoded in a firing sequence $\left(t_{i}^{f}\right)$, where $t_{i}^{f}$ is the firing time of neuron $i$. We will consider here only the first spike of each neuron. The one-spike simplification is relevant in the context of fast processing, where neurons have time to fire only one spike, or in networks with strong synaptic depression, where the initiation of waves is driven by the first presynaptic spike $[36,37,11]$. The issue of spike propagation has been addressed mainly in two extreme cases. (i) The firing times in the sequence are simply related by the relationship $t_{i+1}^{f}-t_{i}^{f}=1 / c$; a traveling wave of velocity $c$ propagates in the network. Such a wave with a constant delay between the spike timings of two consecutive neurons is referred to as a simple wave. Simple waves have been intensively studied in spatially continuous networks of synaptically coupled spiking neurons $[11,5,6,16,31]$. (ii)

*INRIA Grenoble - Rhône-Alpes, Laboratoire Jean Kuntzmann, Inovallée 655 Avenue de l'Europe, Montbonnot, 38334 Saint Ismier, France (arnaud.tonnelier@inria.fr). 
In the synchronous propagation of spiking activity, the sequence $\left(t_{i}^{f}\right)$ is made of successive groups of nearly identical firing times. Such a propagation occurs in layered feedforward networks, the so-called synfire chains [1], and numerical simulations show that stable waves can propagate through the network $[4,10,26]$.

A full synchrony is an extreme case of spike arrangement, and the propagation of firing patterns with a detailed temporal structure has hardly been addressed. Let us assume that information on a sensory stimulus is encoded in an interspike interval, $\delta$. Therefore, the basic problem is the following: how can $\delta$ be transmitted by a spiking neural network ? Trivially, a spiking network composed of two nonconnected transmission lines performs such a processing (see Figure 1.1). A similar synaptic

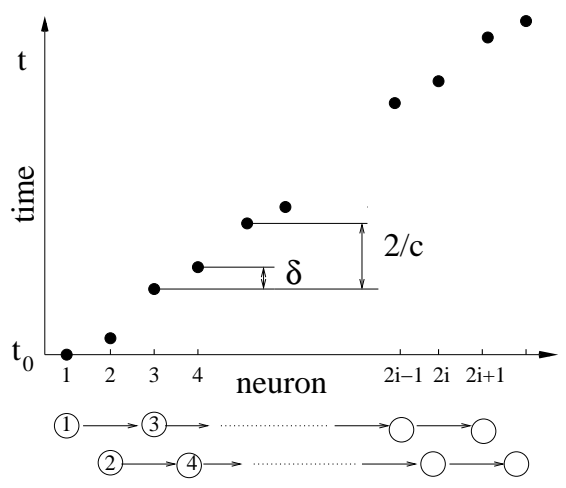

FIG. 1.1. How to transmit an interspike interval $\delta$ ? A trivial solution is a network (bottom of the graph) made of two identical nonconnected transmission lines, where each transmission line supports a traveling wave of velocity $c$. A propagation is initiated in the first transmission line at time $t_{0}$ and in the second one at time $t_{0}+\delta$. The resulting traveling wave conveys the interspike interval $\delta$ at a velocity $c$. The firing times of the neurons are plotted here as solid dots.

architecture combined with coincidence detectors has been used as a crude model of the barn owl's auditory system [24]. The interaural time difference is physically represented through two delay lines and is used to locate a sound with high precision. This specific architecture is highly restrictive and several delay lines have to be used when several interspike intervals have to be transmitted at the same time.

In the present paper, we analyze the capability of a connected ${ }^{1}$ spiking neural network to transmit a spike sequence, making relatively few assumptions on the synaptic architecture. We examine the propagation using highly simplified neuronal model and network connectivity. We consider a spatially structured network of leaky integrateand-fire neurons where the dynamics of the membrane potential of neuron $i$ at time $t, v_{i}(t)$, follows

$$
\frac{d v_{i}}{d t}=-\frac{v_{i}}{\tau_{m}}+I_{i}+I_{\mathrm{app}}
$$

where $\tau_{m}$ is the membrane time constant, $I_{i}$ the total synaptic current for neuron $i$, and $I_{\mathrm{app}}$ is an external drive. When the membrane potential reaches a threshold $\vartheta$ the neuron fires a spike. We consider that each neuron fires only once and we denote by $t_{i}^{f}$

\footnotetext{
${ }^{1}$ the network is said to be connected in the sense of topological space, i.e. there is a path from any neuron to any other neuron in the network
} 
the time at which the neuron located at $i$ fires. When a spike is emitted, the membrane potential is reset to $v_{r}<\vartheta$. This framework allows analytical treatment of the model and provides a first approximation for the study of more complex propagating patterns. Note that a first step towards the investigation of multiple-spike activity propagation has already been achieved in the continuous limit [30].

A change of variables allows us to set $\vartheta=1$ and $\tau_{m}=1$. Therefore, membrane potential and time are dimensionless, and the physical units are retrieved multiplying voltage by a factor of $10 \mathrm{mV}$ and time by a factor of $10 \mathrm{~ms}$ (for instance, a time constant equal to 2 has a physical order of $20 \mathrm{~ms}$ ). In the absence of synaptic input, two distinct regimes exist: when $I_{\text {app }}>1$ the neuron periodically fires, and for $I_{\text {app }}<1$ the neuron requires inputs to fire. We consider here the excitable regime only, and we take $I_{\text {app }}=0$. A presynaptic spike induces a postsynaptic current (PSC) that is described by a so-called $\alpha$-function, $\alpha(t)$. The total synaptic current is taken to be of the form

$$
I_{i}(t)=g_{\mathrm{syn}} \sum_{j} w_{i j} \alpha\left(t-t_{j}^{f}\right),
$$

where $g_{\mathrm{syn}}$ is the total synaptic conductance and $w_{i j}$ is the strength of the synaptic connection from neuron $j$ to neuron $i$. For the network connectivity, we use an infinite chain of neurons, $i \in \mathcal{Z}$, and we assume only that the synaptic strength depends on the 'distance' between neurons, $w_{i j}=w(|i-j|)$, where $w$ denotes the synaptic footprint shape and |.| is the absolute value. For convenience we define $w_{j}=w_{i, i-j}$, and weights are normalized so that $\sum_{j}\left|w_{j}\right|=1$. The rapid processing treatment achieved through the first-spike strategy provides evidence for a feedforward architecture [36] and we take $w_{j}=0$ for $j \leq 0$. We denote by $N$ the number of presynaptic neurons. It should be noted that our architecture significantly differs from synfire chain, where groups of neurons are specifically connected to form successive pools. Here, we focus on a regular synaptic organization derived from a one-dimensional structure. Twodimensional (2D) networks exhibit similar activity but have a lot of paths between two given neurons that make the propagation difficult to analyze especially due to the interplay between paths. In the 1D connectivity, the signal propagation has a limited pathway [12] along a 1D waveguide allowing a characterization of the key parameters that control propagation.

The kinetics of synaptic currents play a fundamental role in spike propagation [13]. To efficiently control the shape of the PSC, we consider the normalized piecewise linear $\alpha$-function

$$
\alpha(t)=\frac{2}{\tau_{r}+\tau_{d}}\left\{\begin{array}{cl}
t / \tau_{r} & , 0 \leq t \leq \tau_{r} \\
1+\left(\tau_{r}-t\right) / \tau_{d} & , \tau_{r} \leq t \leq \tau_{r}+\tau_{d} \\
0 & , \text { otherwise }
\end{array}\right.
$$

where $\tau_{r}$ is the synaptic rise time and $\tau_{d}$ the synaptic decay time. However, the theoretical analysis of spike propagation is done using an arbitrary $\alpha$-function and the piecewise linear approximation is introduced to go further in the analysis and to perform numerical simulations.

Writing the reset condition in $(1.1)$ as the reset current $I_{r, i}=-\left(1-v_{r}\right) \delta\left(t-t_{i}^{f}\right)$, where $\delta$ is the Dirac function, and integrating (1.1) gives

$$
v_{i}(t)=\eta\left(t-t_{i}^{f}\right)+g_{\mathrm{syn}} \sum_{j=1}^{N} w_{j} \epsilon\left(t-t_{i-j}^{f}\right),
$$


where $\eta(t)=\left(v_{r}-1\right) e^{-t} \Theta(t)$ is the reset pulse and

$$
\epsilon(t)=\int_{0}^{t} \alpha(s) e^{-(t-s)} d s \Theta(t)
$$

is the postsynaptic potential (PSP) developed in the neuron as a response to the postsynaptic current (PSC). The function $\Theta$ is the Heaviside step function. Alternatively, the PSP can be defined as the solution of the equation

$$
\epsilon^{\prime}(t)+\epsilon(t)=\alpha(t)
$$

and $\epsilon(0)=0$. Depending on the synaptic time constants, $\tau_{r}$ and $\tau_{d}$, a wide range of PSP kinetics are generated (see Figure 1.2).

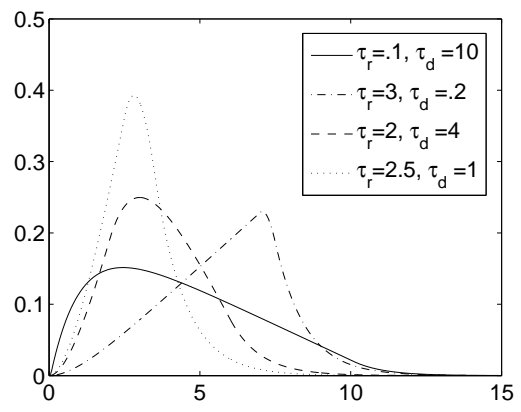

FIG. 1.2. Plot of PSPs for different synaptic time constants. A large variety of PSP kinetics are obtained using long or short rise time combined with long or short decay time.

The paper is organized as follows. In section 2 we develop the theoretical framework for the study of spike propagation. In section 3 we focus on the propagation of a single interspike interval. Numerical simulations on larger sequences with realistic conditions on model parameters are done in section 4 . We finish with a discussion in section 5 .

2. Simple and composite traveling waves. Let us assume that a stimulation initiates in the network a precisely timed sequence of $p$ interspike intervals. The sequence propagates in the network if there exists a velocity $c$ such that the firing times of neurons are given by

$$
t_{p i+k}^{f}=(p i+k) / c+\delta_{k},
$$

where $i$ is the index of the $i$ th repetition of the sequence, $p$ is the sequence length, $k \in\{0, \ldots, p-1\}$ is the label of the successive neurons in the sequence and, $\delta_{k}$ are the propagated interspike intervals, where $\delta_{0}=0$. The traveling wave that propagates the sequence has a spatiotemporal periodicity: during propagation the sequence of spikes is replayed with the same temporal structure along the network as shown in Figure 2.1. Space is divided into 'spatial units' of width $p$, i.e., made of a fixed number, $p$, of associated neurons. We will name these waves composite waves to stress that the propagation can be thought of as the superimposition of several simple waves propagating with the same velocity but with different delays. It should be noted that 


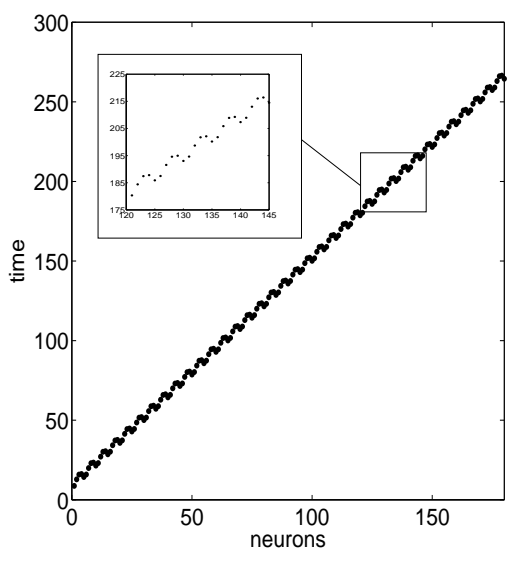

FIG. 2.1. Propagation of a composite wave in a spiking neural network. A packet of five precisely timed spikes is transmitted (see the inset). The firing times are plotted as solid dots.

the effective interspike interval between the two consecutive neurons, $k$ and $k+1$, within a sequence is $I S I_{k}=\delta_{k+1}-\delta_{k}+1 / c$. For convenience, instead of $I S I_{k}$, we use the quantity $\delta_{k}$ that physically measures the deviation from the simple wave obtained for $\delta_{k}=0$ for all $k$. Recall that we consider only the first spike of each neuron and therefore all the indexes used in the present study $(i, p, k)$ are related to a spatial location and do not describe the successive spikes of a neuron that may appear when considering multiple-spike wave propagations [30].

In the following section we focus on simple waves where analytical results on the wave speed are derived and illustrated using different synaptic weight distributions. Stability analysis is also presented. Next, in section 2.2, we provide a framework for the study of composite waves including their stability analysis.

2.1. Simple waves. Simple waves are obtained when $\delta_{k}=0$ or equivalently $p=1$ in (2.1), i.e., the propagated sequence is composed of one neuron. We look for a simple traveling wave solution of the form $v_{i}(t)=V(t-i / c)$ where $c>0$ is the velocity of the wave. Let $\xi=t-i / c$ be the traveling wave coordinate. Simple wave solutions satisfy

$$
V(\xi)=\eta(\xi)+g_{\mathrm{syn}} \sum_{j=1}^{N} w_{j} \epsilon(\xi+j / c) .
$$

The velocity of the wave is obtained from the threshold condition $V(0)=1$ that yields

$$
g_{\text {syn }} \sum_{j=1}^{N} w_{j} \epsilon(j / c)=1 .
$$

The existence of the corresponding traveling wave requires that the causality criterion

$$
V(\xi)<1 \text { for } \xi<0
$$


be fulfilled.

An important question concerns how the wave speed depends on the parameters of the network. Dependence of the wave speed on the synaptic conductance, $g_{\mathrm{syn}}$, is illustrated Figure 2.2 for different synaptic time constants and synaptic weights. In all cases, there exists a minimal value of the total synaptic conductance, $g_{\mathrm{syn}}^{*}$, from which propagation occurs. The speed at the onset of propagation has a well-defined nonzero minimum. In a purely excitatory network (Figure 2.2(a)-(b)) the speed curve is made of one piece with an upper branch where the wave speed increases with $g_{\text {syn }}$ and tends toward infinity as $g_{\text {syn }}$ grows. In an excitatory-inhibitory network (Figure $2.2(\mathrm{c})-(\mathrm{d}))$ the speed curve is made of several pieces depending on the respective strength and distribution of positive and negative weights. We find two parts in Figure 2.2(c) using a Mexican hat-type connectivity and four parts in Figure 2.2(d) with randomly choosen weights (each componant of the vector $\left(w_{i}\right)_{i=1 . . N}$ is randomly chosen). In both plots (Figure 2.2(c)-(d)), the upper component of the speed curves has a shape similar to the one obtained for the purely excitatory network with, in particular, an unbounded wave speed as $g_{\mathrm{syn}} \rightarrow \infty$ whereas the other components present bounded wave speed values. It is worth noting that, numerically, we observe that the lower branch of each piece of the speed curves, i.e., part of the curves where $d c / d g_{\text {syn }}<0$, are prone to being inadmissible solutions. A necessary condition to satisfy criterion $(2.4)$ is $V^{\prime}(0)>0$, i.e., the threshold is reached from below, which leads to

$$
\sum_{j=1}^{N} w_{j} \epsilon^{\prime}(j / c)>0
$$

Note also that branches where $d g_{\text {syn }} / d c<0$ satisfy

$$
\sum_{j=1}^{N} j w_{j} \epsilon^{\prime}(j / c)<0
$$

which is derived from the differentiation of (2.3).

Without loss of generality we take in the following $g_{\text {syn }}>0$. To gain some insight into the shape of the speed curves, let us consider the simplest case of a network where each neuron has only one presynaptic neuron, i.e., $N=1$. We have $w_{1}=1$ (due to normalization), and therefore we have to solve

$$
\epsilon(1 / c)=1 / g_{\mathrm{syn}}
$$

Qualitatively, from the shape of the postsynaptic potential, $\epsilon$, it is obvious that two solutions are found (for $g_{\text {syn }}$ sufficiently large): one in the rising phase of $\epsilon$ and the other in the decaying phase. The solution in the decaying phase is not admissible since (2.4) is not satisfied. Properties of the admissible solution are studied in the following. Using the piecewise linear expression (1.3) of the postsynaptic current $\alpha$, expression (1.5) gives

$$
(2.6) \epsilon(t)=\frac{2}{\tau_{r}+\tau_{d}}\left\{\begin{array}{l}
0, \quad t<0, \\
\frac{1}{\tau_{r}}\left(t-1+e^{-t}\right), \quad 0 \leq t \leq \tau_{r}, \\
1+\frac{\tau_{r}+1-t}{\tau_{d}}+\frac{1}{\tau_{r}} e^{-t}-\left(\frac{1}{\tau_{r}}+\frac{1}{\tau_{d}}\right) e^{-t+\tau_{r}}, \quad \tau_{r} \leq t \leq \tau_{r}+\tau_{d}, \\
\left(\frac{1}{\tau_{r}}+\frac{1}{\tau_{d}} e^{\left(\tau_{r}+\tau_{d}\right)}-\left(\frac{1}{\tau_{r}}+\frac{1}{\tau_{d}}\right) e^{\tau_{r}}\right) e^{-t}, \quad t>\tau_{r}+\tau_{d}
\end{array}\right.
$$




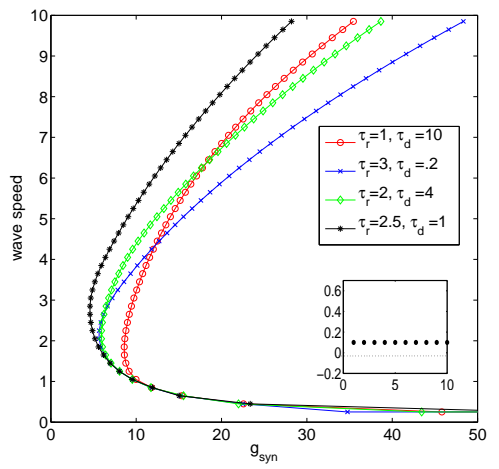

$a$

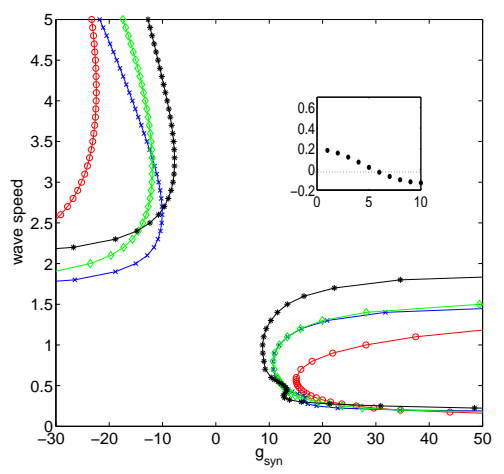

$c$

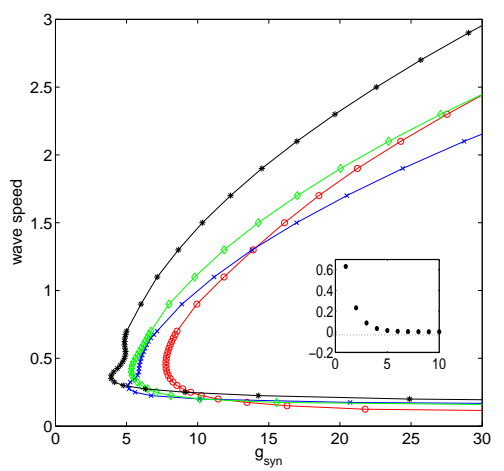

$b$

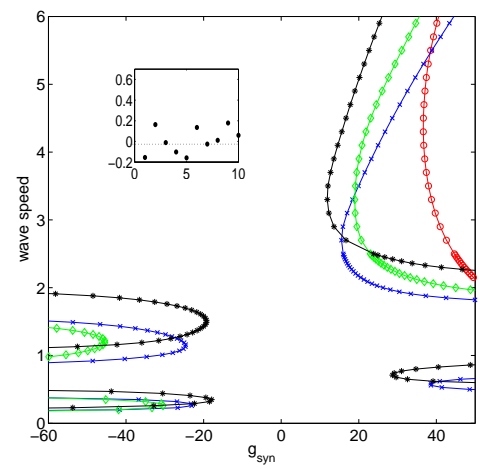

$d$

FIG. 2.2. Simple wave speed as a function of $g_{\mathrm{syn}}$ in a chain of spiking neurons where each neuron has $N=10$ presynaptic neurons. Various synaptic time constants are used : the symbol $\circ$ in red denotes simulations with $\tau_{r}=1, \tau_{d}=2$; the symbol $\times$ in blue is for $\tau_{r}=3, \tau_{d}=0.2$; the symbol $\diamond$ in green is for $\tau_{r}=2, \tau_{d}=4$; the symbol $*$ in black is for $\tau_{r}=2.5, \tau_{d}=1$. We take the following synaptic weights (a) constant, (b) exponential $w_{i}=\exp (-i)$, (c) Mexican-hat (short-range excitation and long-range inhibition for $\left.g_{\mathrm{syn}}>0\right)$, $w_{i}=3 \exp \left(-i^{2} / 50\right)-2 \exp \left(-i^{2} / 162\right)$ and $(D)$ random using an uniform distribution on $(-1 / N, 1 / N)$ ( here, $W=(-0.1551,0.1631,-0.0113,-0.1021,-0.1587,0.1353,-0.0252,0.0112,0.1785,0.0596))$. For each plot the corresponding synaptic weights are shown in the inset.

Thus, the wave speed is obtained by solving the following equations:

$$
\begin{aligned}
x+e^{-x} & =\beta \text { if } & & x \leq \tau_{r}, \\
x+\gamma e^{-x} & =\mu \text { if } & & \tau_{r} \leq x \leq \tau_{r}+\tau_{d},
\end{aligned}
$$

where $x=1 / c$ and

$$
\begin{aligned}
& \beta=1+\frac{\tau_{r}\left(\tau_{r}+\tau_{d}\right)}{2 g_{\mathrm{syn}}} \\
& \mu=1+\tau_{r}+\tau_{d}-\frac{\tau_{d}\left(\tau_{r}+\tau_{d}\right)}{2 g_{\mathrm{syn}}}
\end{aligned}
$$




$$
\gamma=\left(1+\frac{\tau_{d}}{\tau_{r}}\right) e^{\tau_{r}}-\frac{\tau_{d}}{\tau_{r}}
$$

Therefore we have

$$
\begin{aligned}
& c=\frac{1}{W_{0}\left(-e^{-\beta}\right)+\beta}, \quad \tau_{r}^{-1} \leq c, \\
& c=\frac{1}{W_{-1}\left(-\gamma e^{-\mu}\right)+\mu}, \quad\left(\tau_{r}+\tau_{d}\right)^{-1} \leq c \leq \tau_{r}^{-1},
\end{aligned}
$$

where $W_{k}$ denotes the $k$ th branch of the Lambert $W$ function [8]. Expressions (2.7), (2.8) describe the upper branch of the speed curve starting at the knee point

$$
\begin{aligned}
c^{*} & =\left[\tau_{r}+\ln \left(1+\frac{\tau_{r}}{\tau_{d}}\left(1-e^{-\tau_{r}}\right)\right)\right]^{-1}, \\
g_{\mathrm{syn}}^{*} & =\left(\tau_{r}+\tau_{d}\right)\left[2-\frac{2}{\tau_{d}} \ln \left(1+\frac{\tau_{d}}{\tau_{r}}\left(1-e^{-\tau_{r}}\right)\right)\right]^{-1},
\end{aligned}
$$

below which no propagation occurs. Note that the lower branch of the speed curve corresponding to inadmissible solutions is obtained from (2.8), replacing $W_{-1}$ by $W_{0}$. Using the series expansion of $W_{0}$ near the branch point $z=e^{-1}[8]$

$$
W_{0}(z)=-1+\sqrt{2(e z+1)}-\frac{2}{3}(e z+1)+O(e z+1)
$$

we derive, for large $g_{\text {syn }}$ values, the asymptotic behavior of the wave speed

$$
c=\sqrt{\frac{g_{\mathrm{syn}}}{\tau_{r}\left(\tau_{r}+\tau_{d}\right)}}-\frac{1}{6}+O\left(1 / \sqrt{g_{\mathrm{syn}}}\right) .
$$

Let us now generalize the previous asymptotic expansion for $N \geq 1$ presynaptic neurons. For large $g_{\text {syn }}$ values, it is expected that we will find large speed values, i.e., $1 / c \ll 1$. Substituting the Taylor series of $\epsilon$,

$$
\epsilon(u)=\epsilon(0)+u \epsilon^{\prime}(0)+\frac{u^{2}}{2} \epsilon^{\prime \prime}(0)+O\left(u^{3}\right),
$$

into $(2.3)$ and using $\epsilon(0)=\epsilon^{\prime}(0)=0, \epsilon^{\prime \prime}(0)=2 /\left(\tau_{r}\left(\tau_{r}+\tau_{d}\right)\right)$ we find

$$
\frac{g_{\mathrm{syn}}}{c^{2} \tau_{r}\left(\tau_{r}+\tau_{d}\right)}\left(w_{1}+2^{2} w_{2}+\ldots+n^{2} w_{n}\right)+O\left(1 / c^{3}\right)=1 .
$$

It follows that

$$
c^{2} \sim \kappa \sum_{i=1}^{N} i^{2} w_{i}
$$

where the positive constant $\kappa$ is given by

$$
\kappa=\frac{g_{\mathrm{syn}}}{\tau_{r}\left(\tau_{r}+\tau_{d}\right)}
$$

It should be noted that the power law of the velocity as $g_{\text {syn }}$ becomes large is determined by the first nonnull derivative of the PSP taken at $t=0$. From (2.9) we obtain 
the following results for the different synaptic connections.

Constant synaptic weights. For $w_{i}=1 / N$, using $\sum_{i=1}^{N} i^{2}=1 / 6 N(N+1)(2 N+1)$, we obtain

$$
c^{2} \sim \frac{\kappa}{6}(N+1)(2 N+1)
$$

Linearly and quadratically decreasing synaptic weights. For $w_{i}=1 /(|w| i)$, where $|w|$ is the normalization coefficient, we find

$$
c^{2} \sim \frac{\kappa}{2|w|} N(N+1) .
$$

We have $|w|=\gamma+\psi_{0}(N+1)$ where $\gamma$ is the Euler-Mascheroni constant and $\psi_{0}$ is the digamma function (the logarithmic derivative of the gamma function [3]). Note that $|w|$ could be rewritten $|w|=\psi_{0}(N+1)-\psi_{0}(1)$.

For quadratically decreasing weights, $w_{i}=1 /\left(|w| i^{2}\right)$, the asymptotic behavior of the wave speed is given by

$$
c^{2} \sim \frac{\kappa}{|w|} N
$$

The normalization coefficient is $|w|=\frac{\pi^{2}}{6}-\psi_{1}(N+1)=\psi_{1}(1)-\psi_{1}(N+1)$, where $\psi_{1}$ is the trigamma function (the derivative of the digamma function).

Exponential synaptic weights. Let $w_{i}=\exp (-i / \sigma) /|w|$, where $\sigma$ is a positive constant that monitors the synaptic footprint length, and $|w|=z\left(1-z^{N}\right) /(1-z)$ where $z=\exp (-1 / \sigma)$. The analytical expression of the asymptotic behavior of the wave speed is given by

$$
c^{2} / \kappa \sim \sum_{i=1}^{N} i^{2} z^{i}=\frac{z^{N+1}}{(z-1)^{3}}\left(N^{2} z^{2}+\left(1-2\left(N^{2}+1\right)\right) z+(N+1)^{2}\right)-\frac{z(z+1)}{(z-1)^{3}}
$$

For $N$ large, the previous expression simplifies to $c \sim \sqrt{\kappa(1+z)} /(1-z)$.

The accuracy of the different approximations is illustrated Figure 2.3. Numerically, the asymptotic expansions are a good overapproximation of the wave speeds.

2.1.1. Stability of simple waves. Consider a simple traveling wave where neurons successively fire at times $t_{j}^{f}=j / c_{0}$. Suppose that the firing times are perturbed such that $\tilde{t}_{j}^{f}=j / c_{0}+u_{j}$. Asymptotic stability then corresponds to the condition $u_{j} \rightarrow 0$ as $j \rightarrow \infty$.

The perturbed traveling wave solution, $\tilde{v}_{i}(t)$, satisfies

$$
\tilde{v}_{i}(t)=g_{\mathrm{syn}} \sum_{j=1}^{N} w_{j} \epsilon\left(t-(i-j) / c_{0}-u_{i-j}\right) .
$$

Expanding (2.10) to first order in $u_{i}$ yields

$$
\tilde{v}_{i}(t)=v_{i}(t)-g_{\mathrm{syn}} \sum_{j=1}^{N} w_{j} u_{i-j} \epsilon^{\prime}\left(t-(i-j) / c_{0}\right) .
$$




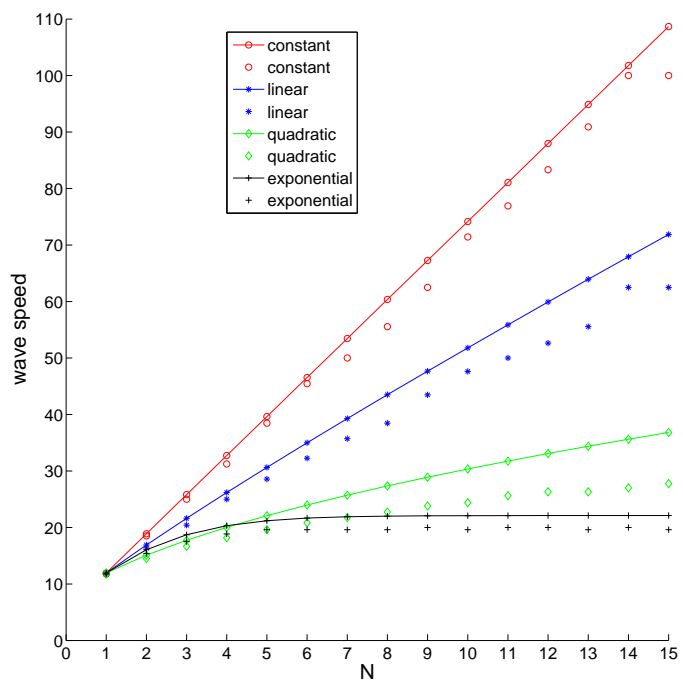

FIG. 2.3. Speed of the simple wave as a function of the number of presynaptic neurons, $N$. The speed obtained with the numerical simulation of a network is compared with the analytical expression derived in the limit of large $g_{\text {syn }}$ values. The lines are for the analytical expression and have to be compared with the corresponding (nonlinked) markers that are for the numerical results. Different synaptic architectures are considered: circles (red) are for constant synaptic weights, stars (blue) are for linearly decreasing weights, diamonds (green) are for quadratically decreasing weights, and crosses (black) are for exponentially decreasing weights. Parameters are $g_{\mathrm{syn}}=30, \tau_{r}=0.1, \tau_{d}=2$. For these parameters, the behavior of the wave speed derived for exponentially decreasing weights as $N$ becomes large is $c \sim 22.1$

Writing the threshold condition at the firing time, $\tilde{t}_{j}^{f}$, leads to the linear equation

$$
\sum_{j=1}^{N} w_{j}\left(u_{i}-u_{i-j}\right) \epsilon^{\prime}\left(j / c_{0}\right)=0 .
$$

Equation (2.11) defines a map that has a general solution of the form $u_{i}=\lambda^{i}$. Substituting this equation into (2.11) yields the characteristic equation

$$
\sum_{j=1}^{N} w_{j}\left(1-\lambda^{-j}\right) \epsilon^{\prime}\left(j / c_{0}\right)=0 .
$$

Les us recall that $\lambda=1$ is a solution because of the translation-invariance property of traveling wave solutions.

Multiplying (2.12) by $\lambda^{N}$, the characteristic equation can be rewritten in the polynomial form

$$
P(\lambda)=\sum_{i=0}^{N} a_{i} \lambda^{i}=0,
$$


where

$$
\left\{\begin{array}{l}
a_{N}=\sum_{j=1}^{N} w_{j} \epsilon^{\prime}\left(\frac{j}{c_{0}}\right), \\
a_{i}=-w_{N-i} \epsilon^{\prime}\left(\frac{N-i}{c_{0}}\right) \text { for } i=0, \ldots, N-1 .
\end{array}\right.
$$

A simple wave is stable if and only if the roots of (2.13) lie in the unit circle. The wave is asymptotically stable if all the roots except the unitary one lie in the interior of the unit circle. Let $Q(\lambda)$ be the polynomial

$$
P(\lambda)=(\lambda-1) Q(\lambda) \text {. }
$$

We have

$$
Q(\lambda)=\sum_{i=0}^{N-1} b_{i} \lambda^{i}
$$

and

$$
b_{i}=-\sum_{k=0}^{i} a_{k}=\sum_{k=N-i}^{N} w_{k} \epsilon^{\prime}\left(k / c_{0}\right) .
$$

where we use the definition (2.14). The simple wave is asymptotically stable if and only if the polynomial $Q(\lambda)$ is Schur stable : all roots of $Q(\lambda)$ lie in the interior of the unit circle. It is worth noting that the Schur stability of polynomials has important applications to the problem of stability and stabilization of discrete time control systems. The so-called Schur-Cohn algorithm has been developped to determine whether all roots of a polynomial are within the unit circle. Here, qualitatively, the stability is determined by the timings of presynaptic spikes with respect to the derivative of the PSP weighted by $w_{j}$. Moreover, a simple sufficient condition for Schur stability is $b_{N-1}>b_{N-2}>\ldots>b_{0}>0$ which gives

$$
w_{i} \epsilon^{\prime}\left(i / c_{0}\right)>0, \quad i \in\{1, \ldots, N\}
$$

Note that $b_{N-1}=-a_{0}-a_{1}-\ldots-a_{N-1}=a_{N}$, and therefore the causality criterion (2.5) leads to the necessary condition of existence $a_{N}>0$ or, equivalently, $b_{N-1}>0$. Condition (2.15) is satisfied in excitatory networks with sufficiently strong coupling. Indeed, for $g_{\text {syn }}$ strong enough, the wave speed is sufficiently large so that the arriving times of the presynaptic spikes, $i / c_{0}$, occur in the rising part of the PSP. This property has interesting connection with the locking theorem stating that oscillations are asymptotically stable if the postsynaptic potential is increasing in time as the neurons fire [14]. Moreover, qualitatively, for moderate values of $g_{\text {syn }}$ it is expected that there exists an index $k$ such that, for $i>k, i / c_{0}$ occurs in the decreasing part of the PSP, $\epsilon^{\prime}\left(i / c_{0}\right)<0$. In this case, it is expected that long-range inhibition plays in favor of the stability of simple waves.

For $N=1$, the traveling wave solutions associated with the upper branch of the speed curve $\left(d c / d g_{\text {syn }}>0\right)$ are stable since the only solution of the eigenvalue problem is $\lambda=1$. Therefore an admissible wave can destabilize only for $N \geq 2$. For $N=2$, we have $Q(\lambda)=b_{1} \lambda+b_{0}$. Thus, the stability is determined by

$$
\lambda=b_{0} / b_{1}=w_{2} \epsilon^{\prime}(2 / c) /\left(w_{1} \epsilon^{\prime}(1 / c)+w_{2} \epsilon^{\prime}(2 / c)\right) .
$$


The simple wave is stable if and only if $|\lambda|<1$. Let us discuss qualitatively the role of the sign of the synaptic weights on the stability of simple waves. From (2.5), the wave is admissible if $w_{1} \epsilon^{\prime}(1 / c)+w_{2} \epsilon^{\prime}(2 / c)>0$. Therefore using (2.16), the necessary condition for the stability of the wave, $\lambda<1$, is rewritten $w_{1} \epsilon^{\prime}(1 / c)>0$. For clarity we assume that the total synaptic input, $g_{\text {syn }}$, is sufficiently strong to have a wave speed such that $\epsilon^{\prime}(1 / c)>0$. Therefore, a local inhibition, $w_{1}<0$ (and therefore $w_{2}>0$, since otherwise the network is purely inhibitory and there is no activity), destabilizes the simple wave and leads to a periodic propagating pattern of two consecutive neurons where one neuron fires and the other stays quiescent. Such a wave is not captured by the study of simple waves and originates from the nonconnected network where $w_{1}=0$ and $w_{2}>0$, a network made of two distinct 1D chains (as shown in Figure 1.1).

2.2. Composite waves. A composite wave is characterized by a spatiotemporal periodicity leading to a recurring pattern of spikes. The trajectories of membrane potentials of neurons within a group are repeated along the network and, during propagation, the network activity is described by

$$
v_{p i+k}(t)=V_{k}\left(t-t_{p i+k}^{f}\right), k \in\{0 \ldots p-1\},
$$

where $i$ is the index of the group, $p$ is the size of the group that is the length of the sequence, $k$ is the index of the neuron within the group and the composite functions $V_{k}$ describe the time course of the $k$ th neuron within the group. Firing times $t_{p i+k}^{f}$ satisfy (2.1). A composite wave is called a $p$-composite wave if $p$ is the period of the spiking activity in the moving wave, i.e, the minimal integer value for which (2.1) is satisfied. The spatiotemporal periodicity of the composite wave yields

$$
t_{j}^{f}-j / c=\tilde{\delta}_{j},
$$

where $\left(\tilde{\delta}_{j}\right)$ denotes the periodic extension of period $p$ of $\left(\delta_{j}\right)$.

Let $\xi=t-t_{p i}^{f}$ be the traveling wave coordinate, where the first neuron in the group is arbitrary choosen as the origin. For convenience, we also define $\xi_{k}=t-t_{p i+k}^{f}$, $k \in\{0, \ldots, p-1\}$, the traveling wave coordinate associated with neuron $k$ within the sequence. We have $\xi_{0}=\xi, \xi_{k}+k / c+\delta_{k}=\xi$. Inserting into (1.4) a solution of the form (2.17) and using the notation previously introduced, the composite function $V_{k}$ satisfies

$$
V_{k}\left(\xi_{k}\right)=\eta\left(\xi_{k}\right)+g_{s y n} \sum_{j=1}^{N} w_{j} \epsilon\left(\xi_{k}+j / c+\delta_{k}-\tilde{\delta}_{k-j}\right) .
$$

The unknown parameters, the velocity $c$ together with the deviations $\delta_{k}$ from the simple wave, are determined from the $p$ threshold crossing conditions that are written

$$
V_{k}(0)=1, k \in\{0, \ldots, p-1\},
$$

where each composite function $V_{k}$ is expressed in its own traveling wave coordinate $\xi_{k}$. We obtain the system

$$
g_{\text {syn }} \sum_{j=1}^{N} w_{j} \epsilon\left(j / c+\delta_{k}-\tilde{\delta}_{k-j}\right)=1, k \in\{0, \ldots, p-1\}
$$


of $p$ equations with $p$ unknowns: $\delta_{1}, \ldots, \delta_{p-1}$, and the velocity, $c$. A solution is said to be admissible if the causality criteria

$$
V_{k}(\xi)<1 \text { for } \xi<0, \quad k \in\{0, \ldots, p-1\}
$$

are satisfied.

The stability analysis of composite waves is presented in the next section. Let us close this section with the following remarks. The destabilization of the simple wave previously discussed for $N=2$ leads to a 2-composite wave that corresponds to the limiting case where $\delta_{1}=\infty$. The composite wave observed Figure 2.1 is a 5 composite wave, i.e., $p=5$, and is supported by a network where each neuron has 10 presynaptic neurons. It should be noted that the number of propagated ISIs is not imposed by the number of presynaptic neurons. For instance, the propagation of a two-spike sequence, shown in Figure 2.4, is also obtained in a network with 10 presynaptic inputs per neuron. This is in contrast to a synfire chain where the size of the propagating sequence is determined by the number of neurons in the successive groups forming the synfire chain.

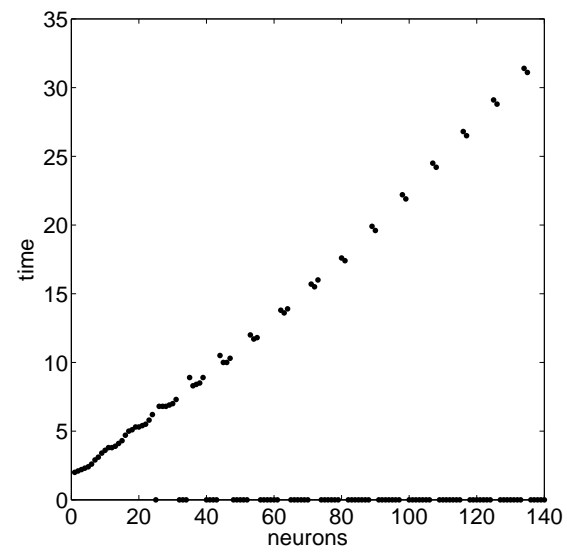

FIG. 2.4. Propagation of a sequence of two spikes in a feedforward network of spiking neurons. After an initial transient, a composite wave made of a packet of 10 neurons propagates. In each packet only two neurons are active, while the others are quiescent ( $a$ dot is placed at time $=0$ to indicate quiescent neurons).

2.2.1. Stability of composite waves. The stability analysis of composite waves is a generalization of the method used for the stability of simple waves and proceeds along the same lines. We sketch here the main steps of the general analysis, and more details will be given in section 3, where the stability of the propagation of an interspike interval is studied in depth. Consider a composite wave that propagates in the network a firing sequence of length $p$. Neurons successively fire at times $t_{p i+k}^{f}=(p i+k) / c_{0}+\delta_{k}$, where $k \in\{0, \ldots, p-1\}$. Let

$$
\tilde{t}_{p i+k}^{f}=(p i+k) / c_{0}+\delta_{k}+u_{p i+k}
$$


be the perturbed firing times. Substituting this ansatz into (1.4) and using the first order expansion of the PSP, $\epsilon(t)$, we obtain

$$
\tilde{v}_{p i+k}(t)=v_{p i+k}(t)-\sum_{j=1}^{N} w_{j} u_{p i+k-j} \epsilon^{\prime}\left(t-t_{p i+k-j}^{f}\right)
$$

where $\tilde{v}_{p i+k}$ is the membrane potential of the perturbed composite wave and $v_{p i+k}(t)$ is the unperturbed solution. Writing the threshold crossing at the perturbed firing time $\tilde{t}_{p i+k}^{f}=t_{p i+k}^{f}+u_{p i+k}$, and after linearization, we end up with a linear system of equations

$$
\sum_{j=1}^{N} w_{j}\left(u_{p i+k}-u_{p i+k-j}\right) \epsilon^{\prime}\left(j / c_{0}+\delta_{k}-\tilde{\delta}_{k-j}\right)=0
$$

that defines $p$ maps $\left(u_{p i+k}\right)_{i}$ for $k=0, \ldots, p-1$. Note that we use $(\tilde{\delta})$, the periodic extension of $(\delta)$ previously defined. Considering the successive firings of neurons during the propagation and due to the nonsymmetric nature of composite waves (with respect to simple waves), we look for a solution of the form

$$
u_{p i+k}=\lambda_{1}^{i+1} \ldots \lambda_{k}^{i+1} \lambda_{k+1}^{i} \ldots \lambda_{p}^{i},
$$

where $\lambda_{l} \in C, l=1, \ldots, p$. Asymptotic stability holds if all nonunitary solutions $\left(\lambda_{1}, \ldots, \lambda_{p}\right)$ satisfy

$$
\left|\prod_{l=1}^{p} \lambda_{l}\right|<1
$$

Inserting (2.21) into (2.20) and multiplying each equation by the appropriate monomial allows us to transform (2.20) into a system of multivariate polynomial equations. More details are given in the next section where the study is extended in two directions. First, we consider the propagation of a single ISI $(p=2)$; second, we study the three nearest-neighbor coupling network $(N=3)$.

3. Propagation of an ISI. A simple wave communicates a single timing, the inverse of the wave speed, and is therefore a poor means of transmiting information about the stimulus that initiates it. Basically, 'all-or-none' information is retained: there is a propagation or a failure. A composite wave conveys more information, and, for instance, a very short ISI can be transmitted. We will say in the following that an ISI is transmitted if there exists $k, \delta_{k} \neq 0$ (even if a simple wave transmits an ISI that is the inverse of the wave speed). Critical questions are (i) how is the ISI related to the initiating stimulus ? (ii) How is the conveyed ISI determined by the connectivity and the synaptic time constants of the network? (iii) What is the relationship between the velocities of composite wave and simple wave? (iv) Is there multistability of the system?

An ISI is transmitted along the network or, equivalently, a 2-composite wave propagates, if there exist two subthreshold time courses of the membrane potential, $V_{1}$ and $V_{2}$, such that

$$
v_{2 i}(t)=V_{1}\left(t-t_{2 i}^{f}\right), v_{2 i+1}(t)=V_{2}\left(t-t_{2 i+1}^{f}\right)
$$


where the two successive firing times $t_{2 i}^{f}, t_{2 i+1}^{f}$ are given by

$$
t_{2 i}^{f}=\frac{2 i}{c}, \quad t_{2 i+1}^{f}=\frac{2 i+1}{c}+\delta,
$$

where $c$ is the wave speed and $\delta$ is the transmitted timing. Note that, strictly speaking, the network propagates two ISIs: $1 / c+\delta$ and $1 / c-\delta$. The two parameters $c$ and $\delta$ are obtained from the threshold crossing conditions which give

$$
\begin{aligned}
& g_{\text {syn }} \sum_{k=1}^{N} w_{k} \epsilon\left(k / c-s_{k}\right)=1, \\
& g_{\text {syn }} \sum_{k=1}^{N} w_{k} \epsilon\left(k / c+s_{k}\right)=1
\end{aligned}
$$

where $s_{k}=0$ if $k$ is even and $s_{k}=\delta$ if $k$ is odd. A 2-composite wave exists if two successive ISIs are different, i.e. $\delta \neq 0$. The time course of the two composite functions $V_{1}$ and $V_{2}$ is given by

$$
\begin{aligned}
& V_{1}\left(\xi_{1}\right)=\eta\left(\xi_{1}\right)+g_{\mathrm{syn}} \sum_{k=1}^{N} w_{k} \epsilon\left(\xi_{1}+k / c-s_{k}\right), \\
& V_{2}\left(\xi_{2}\right)=\eta\left(\xi_{2}\right)+g_{\mathrm{syn}} \sum_{k=1}^{N} w_{k} \epsilon\left(\xi_{2}+k / c+s_{k}\right) .
\end{aligned}
$$

Both wave expressions are given in their respective traveling wave coordinate $\xi_{1}, \xi_{2}$, where we have $\xi_{1}=\xi_{2}+1 / c+\delta$.

For the study of the stability of 2-composite waves, the perturbation of firing times takes the form $\tilde{t}_{2 j}^{f}=2 j / c_{0}+u_{2 j}$ and $\tilde{t}_{2 j+1}^{f}=(2 j+1) / c_{0}+\delta_{0}+u_{2 j+1}$. Using (2.20) with $p=2$, the sequences $\left(u_{2 j}\right)$ and $\left(u_{2 j+1}\right)$ satisfy the following linear equations:

$$
\begin{aligned}
\sum_{j=1}^{N} w_{j}\left(u_{2 i}-u_{2 i-j}\right) \epsilon^{\prime}\left(j / c_{0}-s_{j}\right) & =0, \\
\sum_{j=1}^{N} w_{j}\left(u_{2 i+1}-u_{2 i+1-j}\right) \epsilon^{\prime}\left(j / c_{0}+s_{j}\right) & =0 .
\end{aligned}
$$

Substitution of a solution of the form $u_{2 p}=\lambda_{1}^{p} \lambda_{2}^{p}$ and $u_{2 p+1}=\lambda_{1}^{p+1} \lambda_{2}^{p}$ yields the characteristic equations

$$
\sum_{p=0}^{\left[\frac{N-1}{2}\right]} w_{2 p+1}\left(1-\lambda_{1}^{-p} \lambda_{2}^{-p-1}\right) \epsilon^{\prime}\left((2 p+1) / c_{0}-\delta\right)+\sum_{p=1}^{\left[\frac{N}{2}\right]} w_{2 p}\left(1-\lambda_{1}^{-p} \lambda_{2}^{-p}\right) \epsilon^{\prime}\left(2 p / c_{0}\right)=0,
$$

$$
\sum_{p=0}^{\left[\frac{N-1}{2}\right]} w_{2 p+1}\left(1-\lambda_{1}^{-p} \lambda_{2}^{-p}\right) \epsilon^{\prime}\left((2 p+1) / c_{0}+\delta\right)+\sum_{p=1}^{\left[\frac{N}{2}\right]} w_{2 p}\left(\lambda_{1}-\lambda_{1}^{-p+1} \lambda_{2}^{-p}\right) \epsilon^{\prime}\left(2 p / c_{0}\right)=0,
$$

where $[x]$ is the integer part of $x$. The 2 -composite wave is asymptotically stable if all non-unitary solutions $\left(\lambda_{1}, \lambda_{2}\right)$ of $(3.2)$ satisfy $\left|\lambda_{1} \lambda_{2}\right|<1$. 
Let us now investigate the simplest synaptic architecture that supports the propagation of a 2-composite wave. We consider an excitatory network where each neuron has $N$ presynaptic connections. For $N=1$ the existence of a composite wave is given by the two threshold conditions $g_{\mathrm{syn}} \epsilon(1 / c-\delta)=1$ and $g_{\mathrm{syn}} \epsilon(1 / c+\delta)=1$, together with the causality conditions $g_{\mathrm{syn}} \epsilon(\xi+1 / c-\delta)<1$ and $g_{\mathrm{syn}} \epsilon(\xi+1 / c+\delta)<1$ for all $\xi<0$. The case $\delta=0$ is associated with the simple traveling wave, and we investigate $\delta \neq 0$. We have to solve

$$
\epsilon(1 / c-\delta)=\epsilon(1 / c+\delta) .
$$

The PSP $\epsilon$ is a continuous function that starts at 0 reaches its peak value and goes back to 0 . It thus is possible to find only two distinct solutions, $x, y$, such that $\epsilon(x)=\epsilon(y)=1 / g_{\text {syn }}$ for $1 / g_{\text {syn }}$ smaller than the maximum of $\epsilon$. Clearly, these solutions satisfy $g_{\text {syn }} \epsilon\left(x^{-}\right)<1$ and $g_{\text {syn }} \epsilon\left(y^{-}\right)>1$, where $\epsilon\left(x^{-}\right)=\lim _{u \rightarrow x, u<x} \epsilon(u)$. Then solution $y$ is not admissible since $V_{2}(\xi)=\eta(\xi)+g_{\mathrm{syn}} \epsilon(\xi+1 / c+\delta)$ reaches the threshold from above; i.e., $V_{2}\left(0^{-}\right)>1$.

For $N=2$, we have to solve

$$
\begin{aligned}
& w_{1} \epsilon(1 / c-\delta)+w_{2} \epsilon(2 / c)=1 / g_{\mathrm{syn}} \\
& w_{1} \epsilon(1 / c+\delta)+w_{2} \epsilon(2 / c)=1 / g_{\mathrm{syn}}
\end{aligned}
$$

We find (3.3), and a possible solution is the degenerated composite waves obtained as $\delta \rightarrow+\infty$ where the velocity is given by $g_{\mathrm{syn}} w_{2} \epsilon(2 / c)=1$. Such solutions are not of interest for ISI propagation, and we consider composite waves with finite $\delta$ that require $\delta<1 / c$ (otherwise $\epsilon(1 / c-\delta)=0$ ). From the shape of the PSP $\epsilon$ we have $\epsilon^{\prime}(1 / c-\delta)>0$ and $\epsilon^{\prime}(1 / c+\delta)<0$ (one solution is in the rising part of the PSP and the other one is in the decaying part). Moreover since $2 / c>1 / c+\delta$ we have $\epsilon^{\prime}(2 / c)<0$, and therefore the wave function $V_{2}$ satisfies

$$
V_{2}^{\prime}(0)=w_{1} \epsilon^{\prime}(1 / c+\delta)+w_{2} \epsilon^{\prime}(2 / c)<0 .
$$

Therefore condition (2.5) is not satisfied-i.e., the composite function $V_{2}$ crosses the threshold before $\xi=0$ - and therefore the solution is not admissible.

The simplest synaptic connectivity that may support the propagation of an ISI is a network where each neuron has $N=3$ presynaptic neurons. From (3.1), we define the two auxiliary functions $f$ and $g$ as follows:

$$
\begin{aligned}
& f(1 / c, \delta)=w_{1} \epsilon(1 / c-\delta)+w_{2} \epsilon(2 / c)+w_{3} \epsilon(3 / c-\delta)-1 / g_{\mathrm{syn}} \\
& g(1 / c, \delta)=f(1 / c,-\delta) .
\end{aligned}
$$

The velocity, $c$, and the transmitted timing, $\delta$, are determined from the threshold conditions that give the equations

$$
f(1 / c, \delta)=0, \quad g(1 / c, \delta)=0 .
$$

An intersection point $\left(1 / c_{0}, \delta_{0}\right)$ of the level curves $(3.7)$ indicates a possible solution and provides the speed, $c$, and the transmitted timing, $\delta$, of a 2-composite wave. However, not every solution of (3.7) is related to a composite wave, and some solutions can reach the threshold before $\xi=0$ and therefore are inadmissible. To obtain a 2composite wave, the following causality conditions have to be fulfilled:

$$
V_{1}(\xi)<1 \quad \text { and } \quad V_{2}(\xi)<1 \quad \forall \xi<0 .
$$


It is obvious from $(3.6),(3.7)$ that if $(c, \delta)$ is a solution, then $(c,-\delta)$ is also a solution. In the following we restrict our attention to the case $\delta>0$.

Taking $N=3$ in (3.2) and multiplying the first equation of system (3.2) by $\lambda_{1} \lambda_{2}^{2}$ and the second one by $\lambda_{1}^{2} \lambda_{2}$, the stability of the 2 -composite wave is determined by studying the multivariate polynomial system

$$
\begin{array}{r}
a_{12} \lambda_{1} \lambda_{2}^{2}+a_{11} \lambda_{1} \lambda_{2}+a_{01} \lambda_{2}+a_{00}=0, \\
b_{21} \lambda_{1}^{2} \lambda_{2}+b_{11} \lambda_{1} \lambda_{2}+b_{10} \lambda_{1}+b_{00}=0,
\end{array}
$$

where we employ the following definitions:

$$
\begin{aligned}
& a_{12}=w_{1} \epsilon^{\prime}(1 / c-\delta)+w_{2} \epsilon^{\prime}(2 / c)+w_{3} \epsilon^{\prime}(3 / c-\delta), \\
& a_{11}=-w_{1} \epsilon^{\prime}(1 / c-\delta), \\
& a_{01}=-w_{2} \epsilon^{\prime}(2 / c), \\
& a_{00}=-w_{3} \epsilon^{\prime}(3 / c-\delta),
\end{aligned}
$$

and $b_{i j}$ is obtained from $a_{j i}$ replacing $\delta$ by $-\delta$. The 2-composite wave is asymptotically stable if every root $\left(\lambda_{1}, \lambda_{2}\right)$ of (3.9) satisfies $\left|\lambda_{1} \lambda_{2}\right|<1$.

Figure 3.1 shows the plot of the level curves $f(\delta, 1 / c)=0$ and $g(\delta, 1 / c)=0$ for a constant synaptic coupling, $w_{i}=1 / 3, i=1,2,3$. There are six intersection points, but only two are physically meaningful, i.e. admissible and stable. One corresponds to a stable simple wave (marked SW) and the other one represents a stable 2-composite wave (marked CW). Figure 3.1 also presents the plots of the traveling wave solutions. The graph of an unadmissible solution where the threshold is crossed before firing (crossing indicated by a circle) is depicted. The propagating pattern associated with the two traveling waves is shown in Figure 3.2, where we numerically simulate a network of 40 neurons. It should be noted that the simple wave is faster than the 2 -composite wave, but the two velocities do not significantly differ.

In the last part of this section, items (A)-(D), some properties of 2-composite waves are analyzed using numerical simulations. Unless stated otherwise, parameters are those of Figure 3.1.

(A) Bistability and basins of attraction. The coexistence observed in Figure 3.2 of a stable simple wave together with a stable composite wave shows the bistability of the system. To understand which pattern is more easily initiated and to explore the link between the propagating activity and the initial stimulus that initiates it, we simulate a network using a current injected into the first three neurons of the network ('left' of the network) such that the first three neurons fire successively at time $t_{0}, t_{0}+\Delta_{1}$, and $t_{0}+\Delta_{2}$ (we take $t_{0}=0$ ). For $\Delta_{1}=1 / c$ and $\Delta_{2}=2 / c$ the stimulation mimics the arrival of a simple wave (with a velocity $c$ ), while for $\Delta_{1}=1 / c \pm \delta$ and $\Delta_{2}=2 / c$ a 2-composite wave is initiated (with a velocity $c$ and a transmitted timing $\delta$ ). Level curves are shown in Figure 3.3(a) and (b) and the corresponding basins of attraction of the waves, in the plane $\left(\Delta_{1}, \Delta_{2}\right)$, are depicted in Figure 3.3(c) and (d), respectively, for different synaptic weights. The location in the plane $\left(\Delta_{1}, \Delta_{2}\right)$ of the values corresponding to the simple wave and the 2-composite wave are shown (denoted by green solid dots, and marked SW and CW, respectively). A simple wave is attracting for an initial stimulus in the black region whereas in the gray region a 2-composite wave is initiated. Propagation failure occurs in the white region. In Figure 3.3(b) and (d), we investigate how 2-composite waves are affected by the coupling function of the network and we consider the following perturbation of the previous synaptic weight distribution: $w_{1}=1 / 3+\alpha, w_{2}=1 / 3$ and $w_{3}=1 / 3-\alpha$, where $\alpha>0$ is 


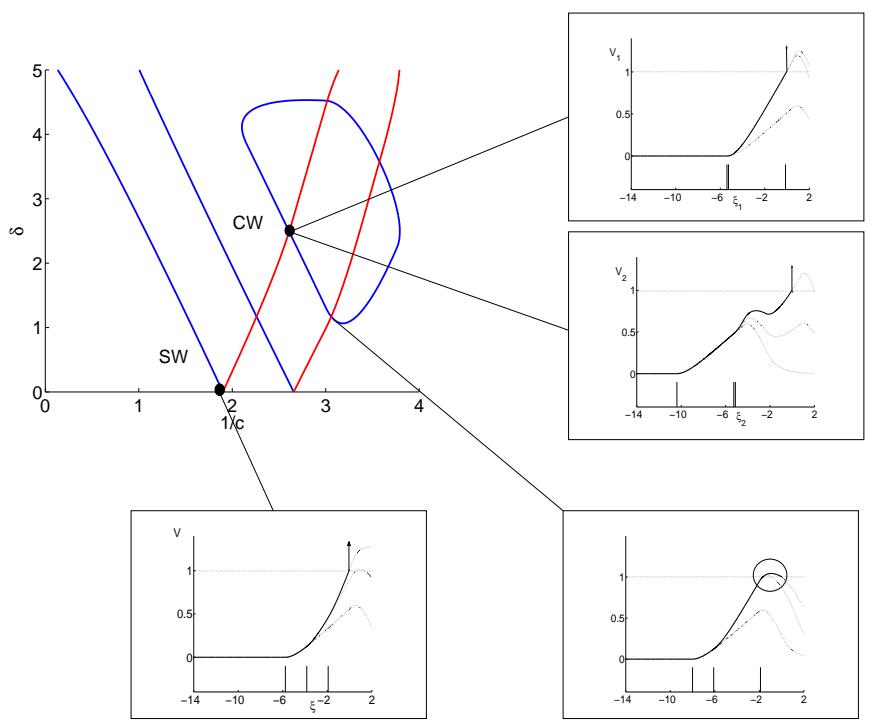

Fig. 3.1. Plot of the level curves $f(1 / c, \delta)=0$ (red curve) and $g(1 / c, \delta)=0$ (blue curve) on the $(1 / c, \delta)$ plane where $c$ is the wave speed and $\delta$ the transmitted timing. The necessary conditions for the existence of waves are fulfilled when the two curves intersect. The intersections emphasized by two black dots denote stable traveling waves: $S W$ stands for a simple wave and $C W$ stands for a 2-composite wave (composed of a sequence of 2 spikes). Other intersections represent unstable or unfeasible waves. The wave functions corresponding to the stable simple wave, the stable 2composite wave (the two composite functions $V_{1}$ and $V_{2}$ ), and an innadmissible wave are depicted (insets) together with the temporal structure of the triplet of presynaptic spikes (vertical bars on the $\xi$-axis). All the induced PSPs are depicted (thin lines) and the total membrane voltage is representedby a solid line. The arrow indicates the postsynaptic spike. Graph is done for $\tau_{r}=6$, $\tau_{d}=2, g_{\text {syn }}=8.4, w_{i}=1 / 3 i=1,2,3$. The stable simple wave has a velocity $c=0.52$. The stable 2-composite wave propagates a time difference $\delta=2.49$ with a velocity $c=0.38$.

a small parameter that controls the deviation from constant synaptic coupling. For $\alpha=0$ synaptic weights are constant. As $\alpha$ increases, the interaction between neurons presents a spatially decaying connectivity. Numerical simulations show that for $\alpha=0.104$ the stable 2 -composite wave disappears. For $\alpha=0.1$, a simple wave with a velocity $c=0.46$ and a 2 -composite wave with $c=0.38$ and $\delta=1.23$, can propagate in the network. The locations of the stable waves are plotted (green dots) together with the location of the unstable waves (red dots marked US).

(B) Wave speeds. The dependence of the wave speeds on the total synaptic conductance, $g_{\mathrm{syn}}$, is illustrated Figure 3.4. There are different critical values of $g_{\mathrm{syn}}$ : the stable and unstable simple waves collapse for $g_{\text {syn }}=7.4$, whereas the stable 2 composite wave disappears near $g_{\mathrm{syn}}=7.0$. Conversely, as $g_{\mathrm{syn}}$ increases, the stable 2 -composite wave vanishes, near $g_{\mathrm{syn}}=9.1$, whereas the simple wave propagates with a velocity $c$ that satisfies the asymptotic law $c=K \sqrt{g_{\mathrm{syn}}}$, as predicted previously. Moreover, we observe the following properties: (i) the composite wave is slower than the simple wave, (ii) velocities of waves and $1 / \delta$ increase with $g_{\mathrm{syn}}$, (iii) there is a bistable regime in which the composite wave coexists with the simple wave, and (iv) there are monostable regimes where a composite wave or a simple wave propagates alone. 


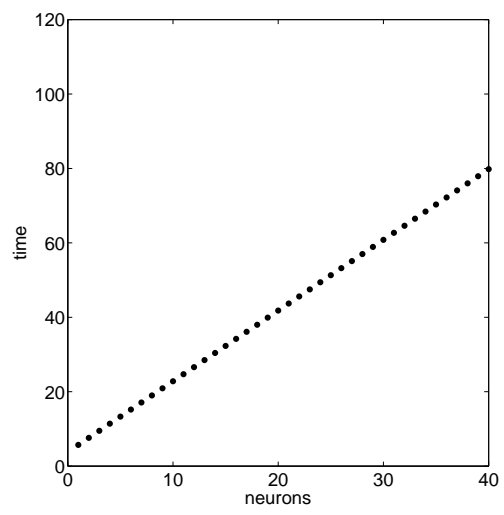

$a$

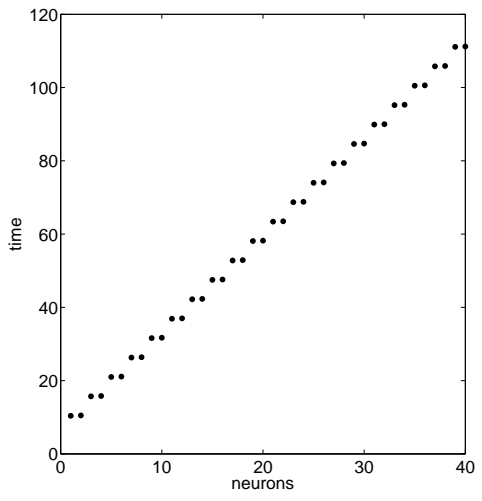

$b$

FIG. 3.2. Bistability between (a) simple wave and (b) 2-composite wave in the network. The dots represent the firing times of neurons. The wave is initiated using a current injected into a group of 3 neurons on the left of the system. In (a) a simple wave propagates, and in (b) a different initial condition leads to the propagation of a 2-composite wave. Here, the 2-composite wave transmits a very short interspike interval (the two corresponding spike timings are almost indistinguable). Parameters are those of Figure 3.1.

(C) Synaptic time constants. To investigate the dependence of the composite waves on the time constants of the network, we compute the locus of existence and stability of 2-composite waves in the $\left(g_{\mathrm{syn}}, \tau_{r}\right)$ plane for different values of $\tau_{d}$ (see Figure 3.5(a)). The synaptic decay time $\tau_{d}$ affects the locus of existence of composite waves but the global shape of the region is preserved. The propagation of composite waves critically depends on $\tau_{r}$ : if $\tau_{r}$ is sufficiently large, i.e., the PSC presents a long increasing period before reaching its peak, a stable composite wave can propagate for appropriate values of $g_{\mathrm{syn}}$, independent of the duration of the decay phase of the PSC, $\tau_{d}$. For large $\tau_{r}$ values, $\tau_{r} \gg 1$, the PSP is triangular, with a rising phase given by

$$
\epsilon(t)=2 t / \tau_{r}^{2}, \quad 0 \leq t \leq \tau_{r} .
$$

Inserting the linear expression of $\epsilon$ (3.10) into (3.5)-(3.6) and assuming that $3 / c+\delta>$ $\tau_{r}$, it is easy to show that the speed, $c$, and the transmitted timing, $\delta$, satisfy $1 / c \simeq \delta$ and $1 / c>\delta$. Therefore, in the traveling wave frame, two spikes arrive simultaneously at $\xi=2 / c$ and one at $\xi=0$ for neurons characterized by the wave function $V_{1}$, and one at $\xi=4 / c$ and two at $\xi=2 / c$ for neurons described by $V_{2}$. This approximation is in good agreement with the numerical simulations depicted Figure 3.5(b) where we find $1 / c=2.99, \delta=2.97$ and, therefore, two of the three presynaptic spikes are fairly indistinguishable (for convenience, we represent them Figure 3.5(b) using a small time delay). The upper branch of the $V$-shaped curve delimiting the existence of 2-composite waves in the $\left(g_{\mathrm{syn}}, \tau_{r}\right)$ plane is related to the minimum value of $g_{\mathrm{syn}}$ for which a 2-composite wave exists. This value is reached when the arrival time of the two nearly equal spikes is precisely the time-to-peak of the PSP, i.e., $\tau_{r}$ in the limit $\tau_{r} \gg 1$. Therefore we have $2 / c \simeq \tau_{r}$ (assumption $3 / c+\delta>\tau_{r}$ is thus a fortiori satisfied), and from (3.5) we find

$$
2 g_{\mathrm{syn}} w \epsilon\left(\tau_{r}\right)=1
$$




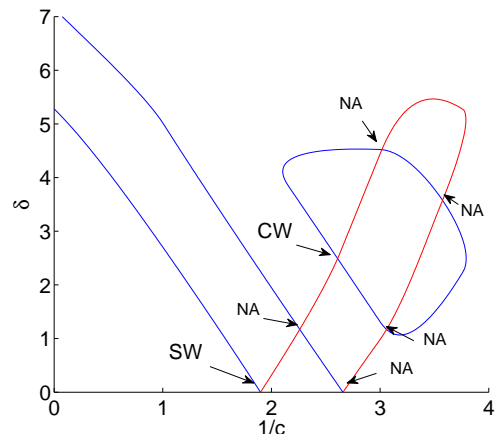

$a$

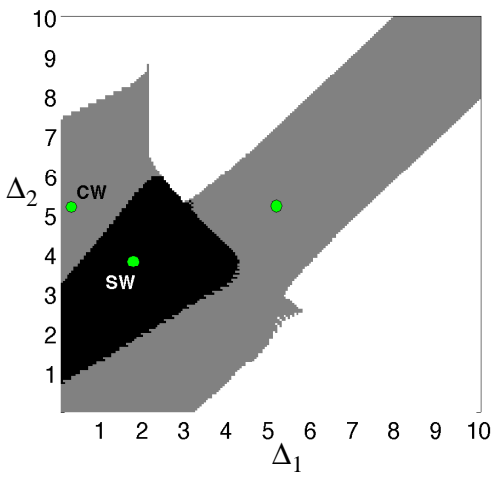

$c$

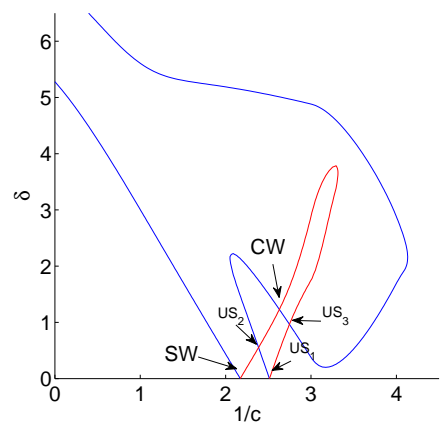

$b$

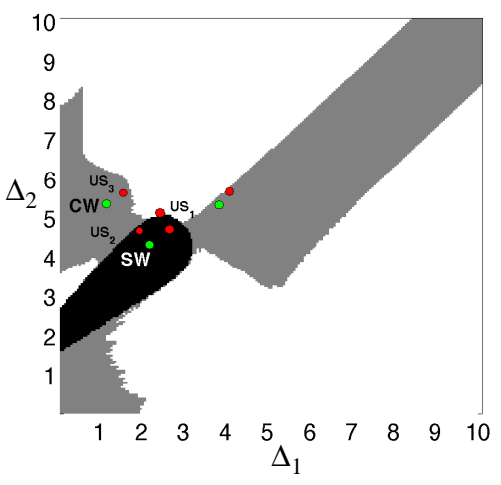

$d$

Fig. 3.3. Plot of the level curves $f(1 / c, \delta)=0$ (red curve) and $g(1 / c, \delta)=0$ (blue curve) for (a) $\alpha=0$ and (b) $\alpha=0.1$. $C W$ denotes the location of a stable 2-composite wave and $S W$ indicates a stable simple wave. US is for an unstable solution and NA for an inadmissible solution. The basins of attraction of the stable propagating waves in the $\Delta_{1}-\Delta_{2}$ plane are shown in (c) and (d) for the stable solutions ( $S W$ and $C W$ ) found in (a) and (b), respectively. In the white region there is no propagation. A stable 2-composite wave is produced in the gray region whereas the black region represents the basin of attraction of the simple traveling wave. The small green dots stand for the precise values of the simple wave (marked $S W$ ) and the 2-composite wave (marked $C W$ ) that are obtained for $\Delta_{1}=1 / c, \Delta_{2}=2 / c$, and $\Delta_{1}=1 / c \pm \delta \Delta_{2}=2 / c$, respectively. Due to the symmetry, the 2-composite wave is associated with a pair of possible values in the $\Delta_{1}-\Delta_{2}$ plane, represented by the two green points with the same ordinate. The locations of the unstable solutions are shown in red in the $\Delta_{1}-\Delta_{2}$ plane: US1 leads to propagation failure, US2 to a simple wave, and US3 to the stable composite wave. Values of time constants are as in Fig.3.1.

for a constant synaptic strength $w_{i}=w=1 / 3$. Using (3.10) we derive the following asymptotic behavior, for $\tau_{r} \gg 1$, of the upper branch of the curve delimiting the region of existence of 2-composite waves:

$$
g_{\mathrm{syn}}=\frac{3}{4} \tau_{r} .
$$

The accuracy of the asymptotic approximation is illustrated Figure 3.5(a). Summarizing, the increasing part of the PSC plays a fundamental role in the existence and stability of 2-composite waves, and 2-composite waves disappear as $\tau_{r}$ becomes too small. Numerical investigations (not shown) suggest that a necessary condition for 


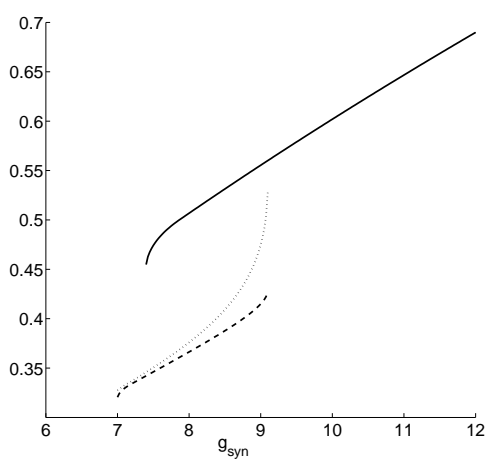

FIG. 3.4. Simple wave speed (solid line) and 2-composite wave speed (dashed line) as a function of $g_{\mathrm{syn}}$. The inverse of the transmitted timing, $1 / \delta$, is also depicted (dotted line). Parameters are those of Figure 3.1.

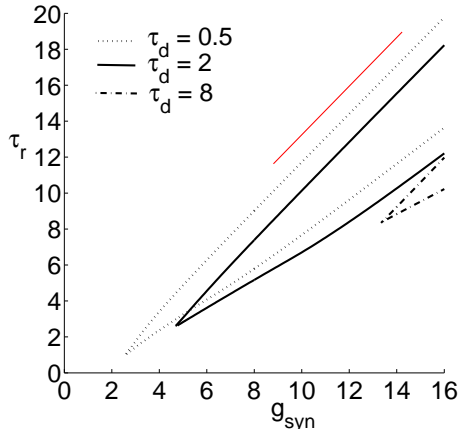

(a)

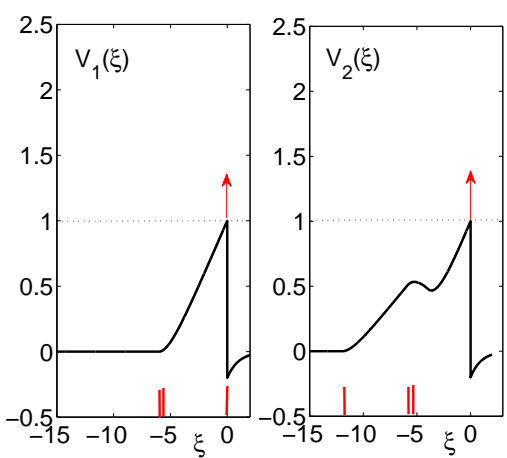

(b)

FIG. 3.5. (a) Locus of existence and stability of 2-composite waves in the $\left(g_{\mathrm{syn}}, \tau_{r}\right)$ parameters plane (region inside the $V$-curves) for different values of $\tau_{d}$. We use $\tau_{d}=0.5, \tau_{d}=2$, and $\tau_{d}=8$. The red straight line denotes the predicted asymptotic behavior of the upper branches of the curves delimiting the locus of existence of stable 2-composite waves. (b) Typical shape of the composite functions, $V_{1}(\xi)$ and $V_{2}(\xi)$, for parameters near the upper branch of the $V$-curves. Spikes are depicted in red. Incoming spikes are plotted as vertical bars on the $\xi$-axis, and the postsynaptic spike is depicted as an arrow. We take $\tau_{d}=2, \tau_{r}=6$, and $g_{\mathrm{syn}}=7.2$.

the existence of a stable composite wave is $\tau_{r}>\tau_{d}$.

(D) Facilitation and depression. Plasticity has a strong impact on the dynamics of neural networks. Long-term plasticity operates at a time scale that is not relevant here since we study one-spike propagation and consider only short-term modifications. Two principal types of short-term plasticity are commonly used: depression and facilitation. Facilitation and depression are presynaptic processes that modify the synaptic changes. Let $s$ be a variable that monitors synaptic changes. The synaptic 
current injected at a neuron $i$ when the presynaptic neuron fires at $t^{f}$ now reads

$$
I_{s}(t)=g_{\mathrm{syn}} s_{i}(t) \alpha\left(t-t^{f}\right),
$$

where $s_{i}$ is a depression, or facilitation, variable which is initially 1 and evolves as $s_{i} \rightarrow \beta s_{i}$ each time a spike is received and

$$
\frac{d s_{i}}{d t}=\frac{1-s_{i}}{\tau_{s}}
$$

at any other time. Depression is obtained for $\beta<1$ and facilitation for $\beta>1$. The time constant $\tau_{s}$ is at a time scale significantly larger than any other one related to the first-spike propagation. Therefore, during the propagation, we neglect the relaxation of $s_{i}(t)$ after the arrival of the traveling wave. In this approximation, the influence of short-term plasticity on the existence of 2-composite waves can be expressed through the functions $f$ of (3.5) and $g$ of (3.6) as

$$
f(\delta, 1 / c)=w_{1} \beta^{3} \epsilon(1 / c-\delta)+w_{2} \beta^{2} \epsilon(2 / c)+w_{3} \beta \epsilon(3 / c-\delta)-1 / g_{\mathrm{syn}}
$$

and $g(\delta, 1 / c)=f(-\delta, 1 / c)$ for $\delta<1 / c$. The influence of facilitation and depression is assessed through parameter $\beta$ and is shown Figure 3.6, where the region of existence and stability of 2 -composite waves is plotted in the $\left(\beta, g_{\mathrm{syn}}\right)$ plane. Networks with either facilitating or depressing synapses support the propagation of 2-composite waves, and, for fixed parameter values, there exists only one stable 2-composite wave. Moreover, there exists a bounded interval of $g_{\text {syn }}$ values where both facilitation and depression allow propagation of composite waves. However, networks with synaptic depression can elicit composite waves for larger $g_{\text {syn }}$ values. In contrast, with facilitation, a 2-composite wave can propagate stably for smaller $g_{\text {syn }}$ values. Moreover, in networks with facilitation, the stable composite wave can coexist with two unstable composite waves, whereas in networks with depression only one unstable composite wave can coexist with the stable one.

4. Biological perspectives. In this section, we numerically study composite waves through the exploration of more realistic biophysical conditions on the model. In the following, unless stated otherwise, the illustrations are done with the numerical simulation of a network in which each neuron receives stimulation from $N=20$ presynaptic neurons.

4.1. Constraints on time constants. One of the critical questions is whether a composite wave can propagate in a network with biologically plausible values of the parameters. As we have shown, in a network with three presynaptic neurons (per neuron), stable composite waves exist but with severe constraints on the time constants of the synaptic currents. In fact, it appears that the constraint $\tau_{d}<\tau_{r}$ has to be fulfilled, which is not physiological and hence unrealistic. However, increasing the number of presynaptic neurons allows us to relax these constraints. This is numerically shown Figure 4.1, where a synchronous sequence of five spikes propagates in a network with the synaptic time contants $\tau_{r}=0.1$ and $\tau_{d}=1$, which correspond to a physical order of $1 \mathrm{~ms}$ and $10 \mathrm{~ms}$, respectively. The simulation of the network is done by imposing the firing time of the 20 first neurons ('left' of the chain). A stimulation that mimics the initiation of a wave of velocity $c$ is done, where $c$ is the approximated value derived previously for the simple wave. Some neurons in the initiation region are blocked randomly. 


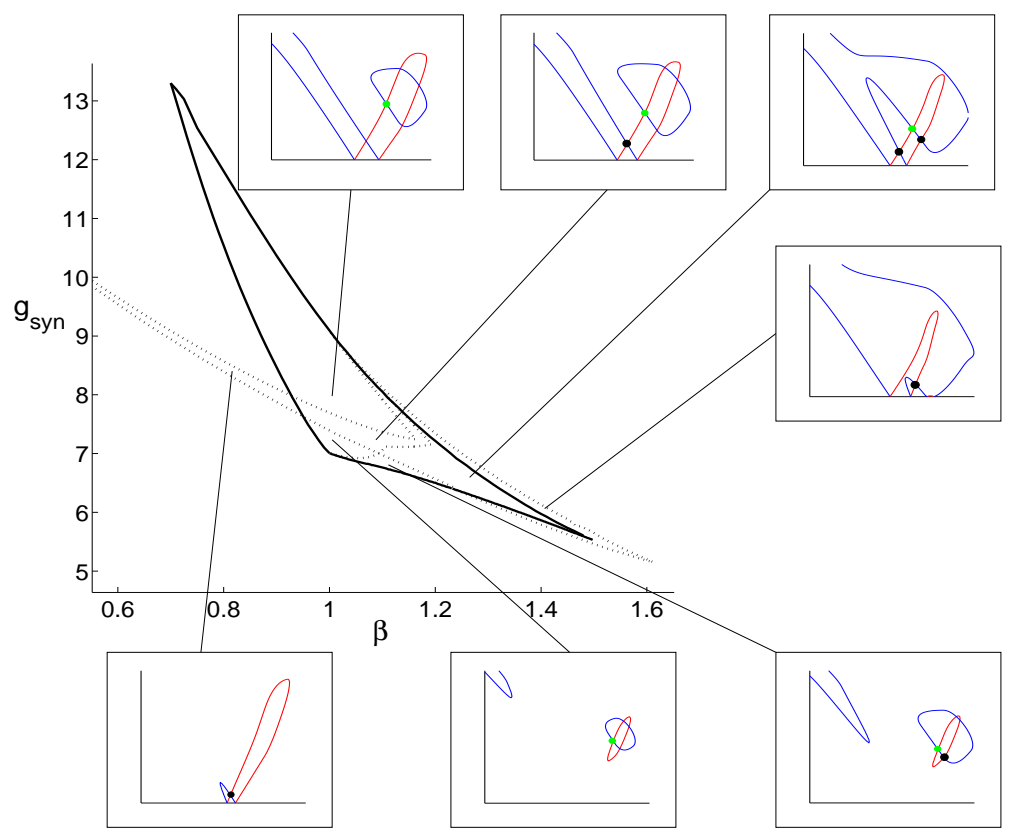

FIG. 3.6. Regimes of existence and stability of 2-composite waves in the $\left(\beta, g_{\text {syn }}\right)$ plane where $\beta$ monitors the facilitation $(\beta>1)$ or the depression $(\beta<1)$ processes of the neurons. Inside the region defined by the solid lines a composite wave exists and is stable. The dotted lines represent the appearance, or the vanishing, of composite waves. The insets give the typical shapes of the levels curves $f(1 / c, \delta)=0$ (red curve) and $g(1 / c, \delta)=0$ (blue curve) in the $(1 / c, \delta)$ plane in the different regions delimited by the dotted and the solid lines. Admissible composite waves are related to the intersection points represented by a filled circle: green circles are for stable solutions and black circles are for unstable ones. Nonadmissible intersection points are not emphasized.

4.2. Robustness of the spike sequence propagation. We have already shown that stable composite waves can propagate through the network and therefore are robust to small deterministic perturbations. However, there are many other sources of variability in neural systems that can affect the transmission of temporally precise sequences of neuronal spikes. Noise is often added in neuronal dynamics to account for the high degree of irregularity observed in in vivo recordings. A simple way to account for variability in spiking neuron model is to consider the following time evolution of the membrane potential

$$
v_{i}(t)=\eta\left(t-t_{i}^{f}\right)+g_{\mathrm{syn}} \sum_{j=1}^{N} w_{j} \epsilon\left(t-t_{i-j}^{f}\right)+X_{i}(t),
$$

where $\left(X_{i}(t)\right)$ are independent random processes that are assumed to be uniformly distributed in $(-\sigma, \sigma)$. This approach is equivalent to a 'noisy threshold' also called the escape model [15]. To assess the robustness of the propagation, a subset of neurons in the chain is affected by the stochastic perturbation of their membrane potential (4.1), and consequently their firing times are affected. We simulate here a chain of 150 neurons and apply the perturbation to neurons from 60 to 90 . For moderate values of $\sigma$ and after a transient, the composite wave is reformed. A typical result is 


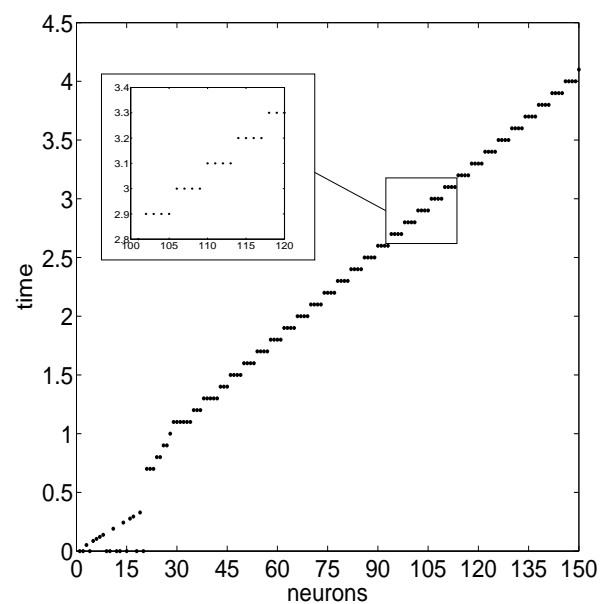

FIG. 4.1. Propagation of a composite wave in a network where each neuron is connected to the $N=20$ left side nearest neurons. After an initial transient, a stable sequence of 4 synchronous spikes propagates. Parameters are $\tau_{r}=0.1, \tau_{d}=1$, and $g_{\mathrm{syn}}=4$. The synaptic weights are constant: $w_{i}=1 / N, i=1, \ldots, N$, where $N=20$.

shown in Figure 4.2(a). Note that one could expect to have, after traversing the noisy region, a propagation failure or the formation of a simple wave. The simulations over repeated trials suggest that the synchronous propagation is very robust to localized noisy threshold perturbations. In Figure 4.2(b) the probability of propagation failure is computed with respect to $\sigma$. The numerical simulations reveal a sigmoid-like shape for the probability of propagation failure of the spike sequence as a function of $\sigma$. For small $\sigma$ values the composite wave propagates (probability of failure near 0 ), whereas large $\sigma$ values lead to a propagation failure. The transition occurs for $\sigma \in(0.4,1.1)$ and follows a linear increase. Recall that the membrane potential has a resting value of $v_{0}=0$ and the threshold, $\vartheta$, has been set to 1 . Therefore $\sigma=0.4$ means a perturbation that could be of an order equal to 40 percent of the total membrane potential amplitude.

It is of interest to note that taking the mean of the firing times over repeated trials for each neuron does not reveal the underlying time structure of the propagating activity as shown Figure 4.3. The reason for this is that the labels of the neurons in the synchronous groups may differ from one trial to another even if a propagating group is still made of four synchronous spikes. This property is related to the translational invariance of traveling waves and has important consequences on the analysis of the multitrial recordings, since such an averaging process destroys the underlying precise sequence propagation.

4.3. A chain of Hodgkin-Huxley neurons. The propagation of a spike sequence has been investigated using a highly simplified neural model. An important issue is the existence of composite waves in more detailed models. To show that composite waves are not inherent to integrate-and-fire models, we simulate a chain of synaptically coupled Hodgkin-Huxley neurons. A trial proceeds as follows. A subset of randomly chosen neurons at the beginning of the network is forced to fire at given 


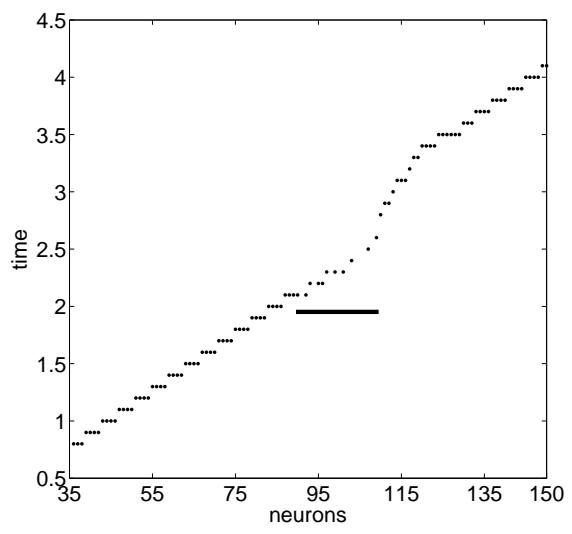

$(a)$

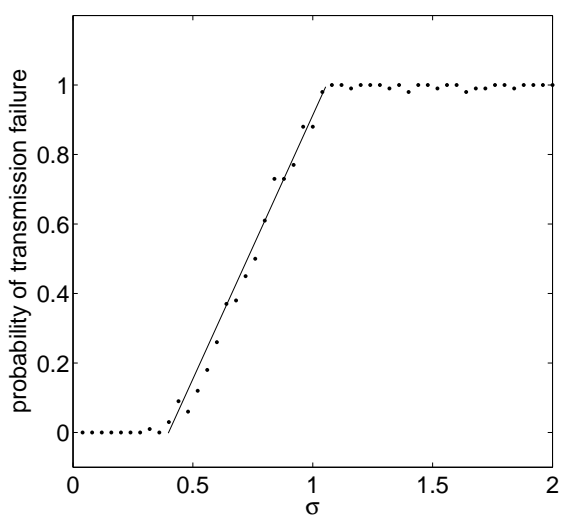

(b)

FIG. 4.2. Robustness of spike sequence propagation. (a) The influence of noise on the propagation of a spike sequence is assessed by applying a stochastic perturbation to the subset of neurons indicated by the straight line $(\sigma=0.3)$. (b) Probability of propagation failure of the spike sequence as a function of $\sigma$. For each point, the probability is computed as a mean over 100 trials. Parameters are those of Figure 4.1. The straight line is not a fit but is only a guide to visualize the linear variation near the transition.

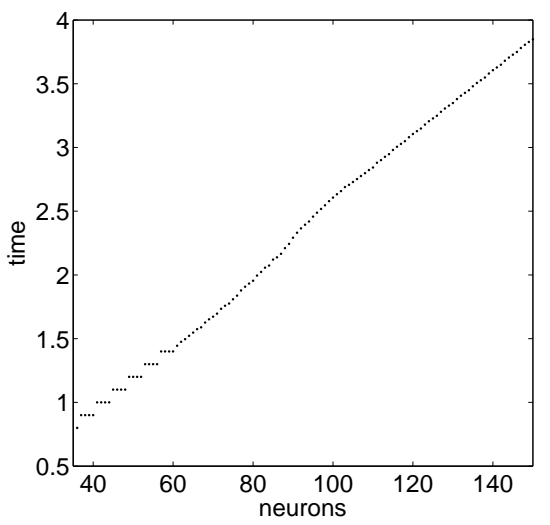

FIG. 4.3. Mean spike times of the neurons across 100 trials. For each individual trial, a group of four synchronous spikes propagates (see Figure 4.2(a)) but the averaging masks the underlying precise temporal structure.

times. The maximal size of the initiating subset is the number of presynaptic neurons in the network, and the firing times of the neurons are chosen so as to mimic the initiation of a simple wave. In order to explore a large panel of initiating patterns of spikes, variability is added through random noise on the imposed firing times. We then investigate the existence of stable composite waves. The numerical simulations (not shown) indicate that precise patterns of spikes propagate in the network. We 
observe that (i) small sequences are more easily initiated (the larger the sequence, the smaller its basin of attraction), and (ii) composite waves that convey synchronous discharges are more robust.

5. Discussion. Repetitions of temporally precise sequences of action potentials have been observed in many brain areas, variously referred to as sequences [29], temporal modules [20], or recurring patterns [35]. Modeling strategies that support precise repetitions of sequences have focused on synfire chains, where spike sequences are generated and propagated along a chain of connected pools. However synfire chains require a highly specific topology in which the propagation is achieved through the synchronous activation of successive pools. In this paper, we use a very simple model, a 1D line of 'one-spike' leaky integrate-and-fire neurons with a spatially homogeneous discrete nearest-neighbor coupling. In line with the results for the spatially continuous approximation, fast simple waves propagate in the network, and, here, we analytically derive some properties of these waves. More interestingly, we find a novel type of traveling waves, the composite waves, i.e., spatiotemporal periodic traveling waves, that support the propagation of precise sequences of spikes. In particular, we show that a time-difference other than the one naturally encoded in the velocity of the wave can be transmitted. Such patterns are an emergent property of the network and do not require conduction delays, unlike the polychronous wavefronts recently studied [21]. It should be noted that spatiotemporal periodic waves have been already reported in spatially continuous network through lurching waves [17, 9, 34]. However, lurching waves differ from composite waves in at least two important ways. First, a lurching wave is characterized by a recruitment cycle that requires inhibition or coupling with large delays, which are not necessary in our network. Second, lurching waves propagate slowly with a characteristic lurching period, unlike composite waves. Here, critical elements for the stable propagation of composite waves are the synaptic time constants, the total synaptic input, and the shape of the synaptic weights. In particular, in networks where neurons have few presynaptic connections, a large synaptic rise time is necessary before composite waves are observed. This requirement vanishes when the number of presynaptic neurons increases.

Spiking neural networks exhibit a large variety of spatiotemporal activities that are, in some still debated manner, involved in the neural processing of information. The selective synchronization of neurons supports the oscillatory coding hypothesis and has been intensively studied. However, the possible role of traveling waves (also reported as correlated activities) in neural coding has been less investigated. Previous theoretical studies have underlined the ability of spatiotemporal sequences of spikes to encode information $[22,23,28]$. One may speculate that the first-spike propagation is involved in fast processing like the fast recognition and categorization of stimuli. Basically, the stimulus-induced propagation along a transmission line implies recognition of the stimulus, and propagation failure leads to nonrecognition. A more elaborate processing requires additional information that may be encoded in the time structure of the propagating sequence. It should be noted that a coding scheme based on traveling waves is independent of how neurons are ordered in the network (unlike the rank order scheme) and has a space-time invariant property: the spike sequence could be 'translated' along the network without changing its information content. We restrict our analysis here to the propagation of one spike along a single channel, but it is expected that several channels and multiple spikes are implied in complex processing. A framework for the study of waves with arbitrary collections of spike times has already been proposed in the continuum approximation [30]. However, the gen- 
eralization to a discrete structure and multiple channels (two-dimensional network) remains to be done. Note that there probably exists a trade-off between the number of presynaptic connections, the number of propagating patterns, and their robustness. One may speculate that sparse coupling leads to the reliable propagation of a limited number of patterns.

During propagation, the activity is characterized by the repetition of the same pattern along the successive groups involved in the transmission. Recurring spatiotemporal patterns have been experimentally observed [29], [35] and are supposed to be involved in a mechanism of learning through spike-time-dependent plasticity. Such a learning role of waves has to be understood in the light of the recently reported developmental role of traveling waves [40].

It is interesting to compare the processing performed by traveling waves with the processing in Hopfield networks [19], where the computation is achieved through the convergence of the underlying dynamical system towards a fixed point. Here, attractors are traveling waves, i.e., spatiotemporal attractors, and the idea of associative memory can be generalized in this context. Challenging problems in understanding the computation made through traveling waves are the estimation of the attraction basins of composite waves, i.e., the link between the initial stimulation and the propagating pattern, and the learning rules that endow the network with composite waves. The ability of spiking neural networks to propagate a spike sequence opens up a whole new range of coding options.

\section{REFERENCES}

[1] M. Abeles, Corticonics, Cambridge University Press, UK, 1991.

[2] M. Abeles, H. Bergman, E. Margalit, and E. Vaadia, Spatiotemporal firing patterns in the frontal cortex of behaving monkeys, J. Neurophysiol., 70 (1993), pp. 1629-1638.

[3] M. Abramowitz, and I.A. Stegun, Handbook of Mathematical Functions, National Bureau of Standards, Applied Math. Series 55, Dover Publications, New York, 1964.

[4] A. Aertsen, M. Diesmann, And M. O. Gewaltig (1996), Propagation of synchronous spiking activity in feedforward neural networks, J. Physiol. Paris, 90 (1996), pp. 243-247.

[5] P. C. Bressloff, Synaptically generated wave propagation in excitable neural media, Phys. Rev. Lett., 82 (1999), pp. 2979-2982.

[6] P. C. Bressloff, Traveling waves and pulses in a one-dimensional network of excitable integrate-and-fire neurons, J. Math. Biol., 40 (2000), pp. 169-198.

[7] S. Coombes, And M.R. Owen, Evans functions for integral neural field equations with Heaviside firing rate function, SIAM J. Appl. Dyn. Syst., 3 (2004), pp. 574-600.

[8] R.M. Corless, G.H. Gonnet, D.E.G. Hare, D.J. Jeffrey and D.E. Knuth, On the Lambert $W$ function, Adv. Comput. Math., 5 (1996), pp. 329-359.

[9] A. Destexhe, T. Bal, D. A. McCormick, and T. J. Sejnowski, Ionic mechanisms underlying synchronized oscillations and traveling waves in a model of ferret thalamic slices, $\mathrm{J}$. Neurophysiol., 76 (1996), pp. 2049-2070.

[10] M. Diesmann, M. O. Gewaltig, And A. Aertsen, Stable propagation of synchronous spiking in cortical neural networks, Nature, 402 (1999), pp. 529-533.

[11] G. B. ERmentrout, The analysis of synaptically generated traveling waves, J. Comput. Neurosci., 5 (1998), pp. 191-208.

[12] O. Feinerman, M. Segal And E. Moses, Signal propagation along unidimensional neuronal networks, J. Neurophysiol., 94 (2005), pp. 3406-3416.

[13] M. Galarreta, and S.Hestrin, Spike transmission and synchrony detection in networks of GABAergic interneurons, Science, 22 (2001), pp. 2295-2299.

[14] W. Gerstner, J. Leo van Hemmen, and J.D. Cowan, What matters in neuronal locking?, Neural Computation, 8 (1996), 1653-1676.

[15] W. Gerstner, and W.M. Kistler, Spiking neuron models, Cambridge University Press, 2002.

[16] D. Golomb, E. Ermentrout, Continuous and lurching traveling pulses in neuronal networks with delay and spatially decaying connectivity, PNAS, 96 (1999), pp. 13480-13485.

[17] D. Golomb, X.J. Wang, And J. Rinzel, Propagation of spindle waves in thalamic slice model, 
J. Neurophysiol., 75 (1996), pp. 750-769.

[18] R.H.R. Hahnloser, A.A. Kozhevnikov, And M.S. Fee, Sleep-related neural activity in a premotor and a basal-ganglia pathway of the songbird, J. Neurophysiol., 96 (2006), pp. 794812.

[19] J.J. Hopfield, Neural networks and physical systems with emergent collective computational abilities, PNAS, 79 (1982), pp. 2554-2558.

[20] Y. Ikegaya, G. Aaron, R. Cossart, D. Aronov, I. Lampl, D. Ferster, and R. Yuste, Synfire chains and cortical songs: temporal modules of cortical activity, Science, 304 (2004), pp. $559-564$.

[21] E. M. Izhikevich, And F. C. Hoppensteadt, Polychronous wavefront computations, Int. J. Bifur. Chaos, 19 (2009), pp. 1733-1739.

[22] D.Z. Jin, Fast convergence of spike sequences to periodic patterns in recurrent networks, Phys. Rev. Lett., 89 (2002), 208102.

[23] D.Z. JiN, Spiking neural network for recognizing spatiotemporal sequences of spikes, Phys. Rev. E, 69 (2004), 021905.

[24] L.A. Jefrress, A place theory of sound localisation, J. Comp. Physiol. Psychol., 41 (1948), pp. 35-39.

[25] R. S. Johansson, ANd I. BiRznieks, First spikes in ensembles of human tactile afferents code complex spatial fingertip events, Nature Neuroscience, 7 (2004), pp. 170-177.

[26] W. M. Kistler, AND W. Gerstner, Stable propagation of activity pulses in populations of spiking neurons, Neural Computation, 14 (2002), pp. 987-997.

[27] G. Laurent, AND H. Davidowitz, Encoding of olfactory information with oscillating neural assemblies, Science, 265 (1994), pp. 1872-1875.

[28] N. Masuda, AND K. Ainara, Spatiotemporal spike-encoding of a continuous external signal, Neural Comp., 14 (2002), pp. 1599-1628.

[29] Z. Nádasdy, H. Hirase, A. Czurkó, J. Csicsvari, and G. Buzsáki, Replay and time compression of recurring spike sequences in the hippocampus, J. Neurosci., 19 (1999), pp. 94979507.

[30] R. Osan, R. Curtu, J. Rubin and G.B. Ermentrout, Multiple-spike waves in a onedimensional integrate-and-fire neural network, J. Math. Biol., 48 (2004), pp. 243-274.

[31] R. Osan And G.B. Ermentrout, The evolution of synaptically generated waves in one-and two-dimensional domains, Physica D, 163 (2002), pp. 217-235.

[32] D.J. Pinto, And B. Ermentrout, Spatially structured activity in synaptically coupled neuronal networks: I. Traveling fronts and pulses, SIAM J. Appl. Math., 62 (2001), pp. 206-225.

[33] D. J. Pinto, R. K. JACKSON, AND C. E. WAYNE, Existence and stability of traveling pulses in a continuous neuronal network, SIAM J. Appl. Dyn. Syst., 4 (2005), pp. 954-984.

[34] J. Rinzel, D. Terman, X.-J. Wang, and B. Ermentrout, Propagating activity patterns in large-scale inhibitory neuronal networks, Science, 279 (1998), pp. 1351-1355.

[35] J. D. Rolston, D. A. Wagenaar, and S. M. Potter, Precisely timed spatiotemporal patterns of neural activity in dissociated cortical cultures, Neuroscience, 148 (2007), pp. 294-303.

[36] S. Thorpe, D. Fize, And C. Marlot, Speed of processing in the human visual system, Nature, 381 (1996), pp. 520-522.

[37] S. Thorpe, A. Delorme, and R. VanRullen, Spike-based strategies for rapid processing, Neural Networks, 14 (2001), pp. 715-725.

[38] W. C. Troy, Traveling waves and synchrony in an excitable large-scale neuronal network with asymmetric connections, SIAM J. Appl. Dyn. Syst., 7 (2008), pp. 1247-1282.

[39] R. VanRullen, R. Guyonneau, and S. Thorpe, Spike times make sense, Trends in Neurosciences, 28 (2005), pp. 1-4.

[40] A. J. Watt, H. Cuntz, M. Mori, Z. Nusser, P.J. Sjöstrom, And M. Häusser, Traveling waves in developing cerebellar cortex mediated by asymmetrical Purkinje cell connectivity, Nature Neuroscience, 12 (2009), pp. 463-473. 